\title{
VALIDACIÓN DE UNA PRUEBA DE HABILIDADES DE PENSAMIENTO PARA ALUMNOS DE CUARTO Y QUINTO DE SECUNDARIA Y PRIMER AÑO DE UNIVERSIDAD
}

\author{
VALIDATION OF A THINKING ABILITIES TEST FOR STUDENTS OF FOURTH AND \\ FIFTH GRADES OF HIGH SCHOOL AND FRESHMEN AT UNIVERSITY LEVEL
}

\author{
Violeta Tapia $M^{1}$, Jorge Luna A. \\ Universidad Nacional Mayor De San Marcos, Perú
}

(RECiBIDO el 01/09/2010, ACEPTAdo el 22/11/2010)

\begin{abstract}
RESUMEN
El presente trabajo da a conocer los resultados de un estudio psicométrico de una prueba elaborada con el fin de evaluar cuantitativa y cualitativamente los procesos cognitivos básicos y superiores del pensamiento, mediante la comprensión lectora. Los dominios que se han tenido en cuenta en este instrumento son el cognitivo y el lingüístico, teniendo como marco teórico de referencia la Teoría de la Modificabilidad Cognitiva Estructural de Reuven Feuerstein, complementada con aspectos de la teoría basada en procesos de Margarita Sánchez; y, la Psicolingüística Textual de T.A.Van Dijk y W. Kintsch.

La muestras seleccionadas corresponden a estudiantes de cuarto y quinto año de educación secundaria y del primer año de estudios universitarios, estatales como particulares de ambos sexos.

El proceso de validación de la prueba comprendió un análisis de la validez de contenido a través de la evaluación por jueces y la evaluación psicométrica realizada en dos fases: en la primera, con una muestra piloto de validación para determinar la confiabilidad de la prueba a través del análisis de dificultad de los ítems, la correlación ítem-test y la estimación del coeficiente de Cronbach. Como resultado de este análisis fueron invalidados trece ítems, de los cuales se eliminaron definitivamente cuatro. La segunda fase consistió en reevaluar los nueves ítems invalidados, previa una revisión y mejora, en una muestra del grupo de estudiantes para la normalización.
\end{abstract}

Un aspecto fundamental de la evaluación psicométrica lo constituyó el Análisis Factorial que permitió fundamentar la teoría en que se basa el instrumento. Se identificaron tres factores subyacentes: inferencia lógica deductiva e inductiva, clasificacione.generalizaciones y razonamiento analógico.

Palabras clave: Habilidades del pensamiento, validez, confiabilidad, análisis factorial.

1 Profesora Emérita de la Universidad Nacional Mayor de San Marcos, Lima Perú. E-mail: violetapiam@yahoo.com 


\begin{abstract}
This paper presents the results of a research study focussing on psychometric testing aimed to evaluate quantitative and qualitative basic cognitive and superior thinking processes during reading comprehension. The cognitive and psycholinguistic domains through reading comprehension were taken in account, based on the theories the Cognitive Structure Modifiability of Reuven Feuerstein, complemented by the process theory of Margarita Sánchez; and the Textual Psycholingüistic processing of T.A Van Dijk y W. Kinysch.
\end{abstract}

The selected samples were taken from male and female students of fourth and fifth grades of High School and freshmen at University level, belonging to public and private institutions.

The result of the analysis showed the content validity of the instrument through expert judgment. The psychometric validation was carried out through two phases: the first one determined the difficulty index of ítems, the reliability through the ítem-test correlation and Cronbach index. After the analysis results, 13 items were invalidated, from which four of them, were definitively eliminated. In the second phase, the remaining nine invalidated items, were reevaluated after a review and improved. The new analysis showed positive results.

An important aspect of the psychometric study was the Factor Analysis that permitted not only to validate the theoretical basis of the instrument, but to identify the underlying factors of the test: logic inference, categorization and analogical reasoning.

Keywords: Thought abilities, validity, reliability, factorial analysis.

\title{
INTRODUCCIÓN
}

En el marco de una nueva línea de investigación sobre el desarrollo de habilidades de pensamiento a través del currículum educativo, el presente trabajo da a conocer los resultados de la validación de un instrumento de evaluación de dichas habilidades cuya elaboración y estandarización en estudiantes de secundaria e ingresantes a la Universidad permitiría no sólo un análisis cualitativo de los procesos cognitivos básicos y superiores del pensamiento sino un nuevo enfoque de análisis de las pruebas de lectura.

En un estudio anterior ${ }^{2}$ se dio a conocer los resultados de un análisis cuantitativo y cualitativo de las preguntas de un texto de lectura y el desempeño lector de un grupo de estudiantes de segundo a quinto grado de Secundaria, en función a las demandas de las operaciones del pensamiento y funciones cognitivas implicadas.

Se trabajó con una prueba de lectura ya estandarizada cuyas preguntas se analizaron previamente con un enfoque cualitativo. Como no era una prueba ad hoc, elaborada para medir específicamente habilidades de pensamiento, sólo se identificaron algunas operaciones básicas: clasificación, inferencia, análisis, síntesis y razonamiento hipotético deductivo.

Los hallazgos de la investigación arrojaron diferencias entre los buenos y deficientes lectores en relación a los procesos cognitivos evaluados, arribándose a conclusiones relevantes que pudieran utilizarse para mejorar la práctica educativa. Sirvieron, asimismo, de base para la construcción de una prueba elaborada con el propósito específico de medir las operaciones del pensamiento de una forma más completa.

2 Tapia, V.; Luna, J. (2008). Procesos Cognitivos y Desempeño Lector" Lima, Perú. Revista de Investigación en Psicología Vol. $11, N^{0} 1$. 
Varias interrogantes giran alrededor de la justificación de la evaluación de las habilidades del pensamiento incluyendo las justificaciones educativas y las bases teóricas y metodológicas para su operacionalización y desarrollo.

Desde lo educativo, las nuevas orientaciones, aprender a aprender de modo eficaz y "aprender a aprender" requieren que los estudiantes dominen y coordinen adecuadamente diversas habilidades, entre ellas las del pensamiento. Ello plantea la necesidad de mejorar el pensamiento en las escuelas a través de instrumentos curriculares y pedagógicos que pongan énfasis no sólo en los contenidos sino en los procesos.

En esta sociedad del conocimiento, los nuevos paradigmas educativos deben apuntar al desarrollo en las personas de un conjunto de competencias como las siguientes:

1. Identificar, reconocer y definir problemas, formular alternativas, solucionar y evaluar resultados.

2. Capacidad de autonomía en la toma de decisiones.

3. Utilizar procesos de pensamiento teórico-abstracto.

4. Pensar estratégicamente, planificar y responder creativamente a demandas cambiantes.

5. Autodisciplina en el trabajo.

6. Alta capacidad de cooperación y liderazgo.

7. Frente al avance de nuevas formas de organización: polivalencia, polifuncionalidad y flexibilidad.

8. Frente a la necesidad de recalificación permanente: capacidad y actitud positiva y, autoaprendizaje continuo.

9. Manejar sus estados afectivos y motivación, tanto como para superar conflictos como para trabajar bajo presión.

10. Aprender a enfrentar una realidad cambiante con principios sólidos y criterios claros y flexibles.

11. Respetar códigos éticos en el marco de una formación en valores.

Diversos estudios han demostrado que el conocimiento se adquiere en diferentes dominios (matemática, ciencias naturales, ciencias sociales, etc.) que presentan características diferenciadas, sin que ello signifique que no se construyan capacidades de índole general. Lo que el sujeto construye son significados, representaciones mentales respecto a los contenidos. Estos dejan de ser vistos como contenidos puramente verbales y son reconocidos como conceptos y operaciones vinculados a la práctica social.

Cada área curricular se caracteriza por un modo particular de discurso, por una manera de plantear los problemas, de ver la realidad, de relacionar, valorar y resolver problemas. Se ha demostrado que es posible desarrollar de manera integral y progresiva las dimensiones cognitivas y retóricas de una disciplina situando las prácticas discursivas de la lectura y escritura en sus ambientes de aprendizaje. 
Las disciplinas son culturas específicas especializadas; cada disciplina constituye una forma particular de ver, de pensar, de experimentar y conocer un sector limitado de lo real y, también una forma específica de hablar y actuar sobre él. Cada asignatura es un sistema de pensamiento que funciona de manera particular, según sus propias reglas (Perkins, 1993, citado por Moreno, 2009).

En educación superior el saber una disciplina es el haber desarrollado la condición de experto en dos dominios: el de las representaciones abstractas (cognitivo) y el proceso de construcción y comunicación de dicho dominio (lingüístico). Estar en la capacidad de utilizar las representaciones abstractas en contextos específicos gracias a que se ha apropiado de las convenciones lingüísticas y retóricas que permitan comprender, explicar, construir y divulgar el conocimiento.

Estos dos grandes dominios, el cognitivo y el lingüístico-retórico en la construcción de los conocimientos curriculares constituyen la justificación teórica de la evaluación psicométrica de las habilidades de pensamiento fundamentadas, en relación al dominio cognitivo, en la Teoría de la Modificabilidad Cognitiva Estructural de Reuven Feuerstein complementada con algunos aspectos de la Teoría basada en procesos de Margarita Sánchez; y, al dominio lingüístico-retórico, en el modelo teórico de procesamiento de textos desarrollado por Walter Kintsch y T.Van Dick.

Uno de los propósitos del presente estudio se relaciona con los aportes que puedan brindar los resultados a las evaluaciones en el aula, en la medida que, generalmente las pruebas psicométricas y estandarizadas han sido de poca utilidad para mejorar las prácticas de enseñanza. El enfoque del análisis cualitativo que complementa el nivel de logro cuantitativo permitirá conocer de forma más específica, dinámica y funcional, el grado de dominio de las habilidades de pensamiento subyacentes en el aprendizaje de las diversas áreas educativas, propiciando en el maestro la toma de conciencia de la importancia de las capacidades que los alumnos deben desarrollar y una apertura a nuevas proyecciones conceptuales en relación a la didáctica y a las formas de evaluar a los alumnos.

\section{OBJETIVOS}

\section{General}

Elaborar y validar una prueba de habilidades de pensamiento a través de la lectura.

\section{Específicos}

1. Determinar la validez de contenido a través del criterio de jueces.

2. Determinar la confiabilidad de la prueba por consistencia interna.

3. Determinar la validez de constructo mediante el análisis factorial.

4. Establecer la consistencia interna de los factores identificados a través de la confiabilidad de los factores identificados. 


\section{Marco Teórico Conceptual}

La fundamentación teórica que apoya los modelos para la evaluación y desarrollo de las habilidades de pensamiento se basa en las teorías sobre el funcionamiento de la mente y la estimulación de los procesos cognitivos que acompañan al acto mental; teorías que provienen de la Psicología Cognitiva y de la Ciencia Cognitiva, siendo los más representativos: H. Gardner $(1983,1985)$; R. Sternberg $(1985,1987)$; Goleman (1986); M. Sánchez $(1985,1992,2000)$ del paradigma de procesos y, R. Feuerstein del modelo Modificabilidad Cognitiva Estructural $(1991,1993,1997)$ que enmarca principalmente el presente estudio.

\section{La Modificabilidad Cognitiva Estructural}

La base teórica del primer dominio (cognitivo) la constituye fundamentalmente la Teoría de la Modificabilidad Cognitiva Estructural, una teoría dinámica de la Inteligencia que la define como la propensión del organismo a ser modificado en sus propias estructuras, como respuesta a la necesidad de adaptación a nuevos estímulos sean de origen interno y externo.

La modificabilidad significa modificación cualitativa de la estructura misma (no sólo mayor cantidad de información), siendo inherente a ella la dinámica y la mecánica de la adaptación. Es la adaptabilidad lo que es inherente a la resolución de problemas y abarca tanto elementos puramente cognitivos como creativos y motivacionales.

Es cognitiva por cuanto la adaptabilidad requiere de nuevas estructuras cognitivas que configuran los elementos afectivos de nuestra personalidad. Reconociendo la estrecha interdependencia entre los factores cognitivos y afectivos para determinar el comportamiento humano, considera la cognición como un factor clave para la adaptación exitosa.

Lo estructural se refiere al mejoramiento global del funcionamiento del individuo. Para ello se requieren tres condiciones: la relación estrecha entre las partes y el todo, la tendencia a implicarse en el procesos de cambio (transformación) y, la autoperpetuación y naturaleza autorreguladora del proceso de cambio.

Dos son los aspectos de la inteligencia humana:

La plasticidad, lo cual lleva a Feuerstein a postular que la modificabilidad es accesible a todos los seres humanos, sin tener en cuenta la etiología exógena o endógena de su condición, su gravedad o la edad. Es una modificación estructural del funcionamiento cognitivo de una persona que refleja ese cambio, apartándose significativamente del curso y dirección del desarrollo previsto genéticamente.

La flexibilidad o capacidad para diversificarse sin perder la continuidad y constancia de la estructura. Capacidad para modificase en algunos puntos críticos de su comportamiento mental, estilo cognitivo o modalidad de interacción.

La mente humana es concebida como un sistema abierto, activo y modificable, constituida por un conjunto de procesos cognitivos o actividades que operan con representaciones 
simbólicas del mundo y que permiten organizar las estructuras del conocimiento. Entre éstos, el pensar es un acto que involucra un constructo complejo y abstract.el pensamient. y como tal, está constituido por otros constructos. "Pensar, en un sentido amplio, es la búsqueda de significados: es un proceso mental por medio del cual el individuo le da sentido a la experiencia. Pensar sirve a muchos fines e involucra multitud de operaciones y tareas mentales, tales como recordar, resolver problemas, tomar decisiones, razonar, entre otros" (Sánchez, 2002).

Mayer (citado por Sánchez, 2002), sugiere una definición general, única de pensar que incluye tres procesos básicos:

1. Pensar es cognoscitivo, se infiere directamente de la conducta, ocurre internamente en la mente o sistema cognitivo de la persona.

2. Pensar es un proceso que involucra manipulación de un conjunto de operaciones sobre conocimiento en el sistema cognoscitivo.

3. Pensar es un proceso dirigido que permite resolver problemas. En otras palabras, pensar es lo que pasa en la mente de un sujeto cuando resuelve problemas, esto es la actividad que mueve al individuo (o trata de moverlo) a través de una serie de etapas o pasos, de un estado dado a uno deseado.

El conocimiento se concibe como un constructo, producto del intelecto humano, que tiene su origen en los referentes del mundo y se construye mediante la interacción de dos tipos de componentes, uno semántico y otro procedimental. El componente semántico provee las estructuras conceptuales y el componente procedimental proporciona los procesos y operaciones del pensamiento. La organización del conocimiento en estructuras depende de su naturaleza semántica o procedimental (Sánchez, 2002).

Las estructuras de conocimiento que se construyen a partir de ciertas reglas de procesamiento en relación a diferentes contenidos (académicos, situaciones de la vida) ponen en juego un conjunto de procesos cognitivos y afectivos, actuando los primeros como operadores intelectuales capaces de transformar los estímulos en representaciones mentales; y los segundos constituyendo el aspecto energético disposicional que motiva a la persona a involucrarse en una actividad mental.

Según la teoría MCE, las operaciones como procesos cognitivos, en términos formales, se refieren a la manipulación y combinaciones mentales de representaciones internas de carácter simbólico (imágenes, conceptos, palabras, etc); es decir, constituyen un conjunto de acciones interiorizadas y coordinadas por las cuales elaboramos información de diferentes fuentes internas y externas.

El término procesos cognitivos se refiere asimismo, a las funciones cognitivas y a las estrategias que realiza la persona que aprende. Para Feuerstein (1979), las funciones cognitivas, como actividades del sistema nervioso explican en parte, la capacidad que tienen las personas para servirse de la experiencia previa en su adaptación a nuevas situaciones. Constituyen el soporte básico de las operaciones que permiten, desde los procesos cognitivos, interiorizar información y autorregular el organismo para facilitar el aprendizaje significativo; son actividades mentales o subestructuras psicológicas que el individuo organiza para enfrentar situaciones tanto de la vida diaria como académica. 
Se agrupan en tres categorías: a) funciones de entrada, las cuales son utilizadas en la búsqueda de información, b) funciones cognitivas de elaboración, utilizadas en el procesamiento de la información; c) funciones cognitivas de salida, que son utilizadas para expresar la respuesta o resultado del acto mental.

La forma de organizar y secuenciar las operaciones da como resultado las estrategias cognitivas y metacognitivas que constituyen un conjunto de acciones internalizadas que el individuo utiliza para procesar información y para controlar y autorregular dicho procesamiento.

Según Pozo (1994), la relación funciones-operaciones-estrategias constituye niveles multidimensionales y dinámicos de un mismo continuo que se van integrando en una cadena de complejidad creciente en los procesos de pensamiento.

La teoría de la MCE, asimismo, identifica y analiza la modalidad de interacción human. entorno, la Experiencia del Aprendizaje Mediado (EAM) como un factor explicativo determinante del cambio estructural de la persona en contraste a la exposición directa a los estímulos.

La Experiencia de Aprendizaje Mediado es ampliamente definida como una cualidad de interacción del ser humano con su ambiente sociocultural que resulta de los cambios introducidos en esta interacción por un mediador humano. El mediador filtra y enmarca el estímulo con el que regula la conducta del niño, joven o adulto. El mediador selecciona, organiza y planifica los estímulos, variando su amplitud, frecuencia e intensidad, y los transforma en poderosos determinantes del comportamiento.

Tres son los criterios de carácter universal para que la intervención sea considerada como EAM:

La mediación de la intencionalidad y la reciprocidad, que significa que el mediador actúa con propósito; su intención consiste en implicar al sujeto en la experiencia de aprendizaje, de modo que es el mediador quien selecciona y organiza la información. Por parte del alumno, la reciprocidad consiste en la creación de un sistema de alerta, una predisposición a seguir la mediación. El proceso es mutuo.

Trascendencia, en el sentido de la mediación, de ir más allá de la necesidad inmediata y situación concreta del momento, es la aplicación del aprendizaje a situaciones nuevas y diferentes. Se amplía el sistema de necesidades y valores; y,

Mediación del significado, factor energético, responsable del comportamiento interactivo. Consiste en presentar las situaciones de aprendizaje de forma interesante y relevante para el sujeto, de manera que se implique activa y emocionalmente en la tarea.

Otras condiciones o parámetros se relacionan directamente con la cultura a la que pertenece el sujeto, como son, entre otros, la mediación del sentimiento de competencia, la regulación y control del comportamiento, la mediación de la individualización y la diferenciación psicológica, de la búsqueda, planificación y logro de objetivos.

Del sistema teórico conceptual se han desarrollado sistemas aplicativos importantes, cuyo eje principal es la EAM, como método fundamental de la intervención que además de 
cumplir un papel explicativo de la MCE es la base del Programa de Enriquecimiento Instrumental (PEI) en la medida que sirve como guía para modelar las interacciones que producirán la modificabilidad y la flexibilidad que son necesarias para la adaptación humana; y, del Modelo de Evaluación Dinámica del Potencial de Aprendizaje (LPAD)

El Programa de Enriquecimiento Instrumental (PEI) es una estrategia de intervención cognitiva multidimensional desarrollada por Feuerstein con el fin de corregir las funciones cognitivas deficientes y afianzar el potencial de aprendizaje de niños a partir de diez años, adolescentes y adultos con necesidades educativas especiales como son:

1. Estudiantes con dificultades generales y específicas de aprendizaje.

2. Estudiantes normales con bajo rendimiento.

3. Estudiantes superdotados.

4. Estudiantes con diferencias culturales.

5. Personas ciegas y sordas.

6. Pacientes con problemas neurológicos y psiquiátricos en programas de rehabilitación.

7. Personas de tercera edad.

La meta principal del PEI es incrementar la propensión del aprendizaje de los estudiantes, equipándolos con los requisitos y procesos de aprendizaje, es decir, aumentar la modificabilidad estructural cognitiva del sujeto retrasado en su encuentro con los estímulos y experiencias de la vida.

La Evaluación Dinámica del Potencial de Aprendizaje ideada por Feuesrstein y colaboradores, es un intento sistemático alternativo al uso de los tests psicométricos, en la que define potencial de aprendizaje, como la capacidad que tienen las personas para pensar y desarrollar conductas inteligentes.

A través del LPAD se extraen conclusiones fundadas en la observación sistemática de determinadas tareas acerca de la naturaleza y adecuación del desarrollo de las funciones cognitivas importantes, la relativa facilidad en que tales funciones pueden modificarse y con qué prontitud se aplican las funciones modificadas a las nuevas tareas.

Este método de evaluación reúne los siguientes criterios:

1. Siguiendo un diseño pre test-entrenamiento-post test, el método trata de introducir cambios a través de la enseñanza y la mediación dentro de la misma situación del test y luego evaluar la actuación modificada y su significado. Se brinda refuerzo y retroalimentación. Los pasos característicos incluyen la medición de los niveles de eficacia con una tarea (línea base), entrenamiento en que se enseñan los principios cognitivos específicos que pueden requerirse en el aprendizaje en sí mismo (experiencia de aprendizaje mediado) y subsiguiente evaluación de la aplicación de los procesos recién adquiridos.

2. Se enfatiza la evaluación del proceso en vez del producto con el presupuesto que la inteligencia no es un objeto a ser medido, sino un proceso a ser evaluado que es dinámico, complejo y multidimensional. 
Existe un intento a revelar la propensión para el cambio cognitivo y no simplemente registrar el nivel de funcionamiento actual. Su finalidad es sugerir formas apropiadas de intervención psicoeducacional y no clasificar.

\section{El modelo teórico del proceso de comprensión lectora}

El modelo teórico del segundo dominio se refiere a los mecanismos y procesos implicados en el procesamiento de textos que explican la precisión y eficacia de la comprensión de los mismos y, a través de los cuales se activan las habilidades de pensamiento.

Partimos del concepto de lectura, actualmente caracterizada como un conjunto de procesos cognitivos y psicolingüísticos, dinámico e interactivo de construcción el significado de un texto (comprensión) en el que se combinan los conocimientos y experiencias previas, la competencia lingüística (fonológica, léxica, sintáctica, semántica), la información aportada por el texto y el contexto (de acuerdo a la gramática textual). Los procesos propiamente cognitivos que participan son los de percepción y reconocimiento de patrones visuales y auditivos específicos, memoria a corto y a largo plazo, y pensamiento (proceso de representación de operaciones de pensamiento y cognición.

En este proceso dinámico e interactivo, el lector crea una relación jerárquica (proposiciones derivadas del texto), unas más generales que otras, relacionando e integrando la información del texto a las estructuras existentes (esquemas), identificando ideas principales, elaborando hipótesis, ejecutando razonamiento hipotético deductivo, realizando inferencias, razonamiento analógico, resumiendo y formando una representación o modelo mental del mismo. Este modelo mental tendría como base las operaciones y funciones del pensamiento que posibilitan el uso de estrategias cognitivas y metacognitivas para una mejor asimilación de la nueva información a la estructura o esquema cognitivo del lector.

Es importante destacar el componente afectiv.motivacional que influye en el interés, propósito, emoción o persistencia a través del cual el lector se compromete con el texto.

Desde la perspectiva sociocultural, se destaca también, por un lado, la importancia del medio social y ambiental en el procesamiento de la lectura, en la medida que se compatibiliza con la cultura del autor o la cultura reflejada en el texto y, por otro, su aprendizaje, a través de interacciones sociales en una variedad de actividades y relaciones en las que juega un rol importante la mediación de los adultos (padres y maestros ) y los pares buenos lectores.

En relación al modelo de procesamiento textual desarrollado por los autores Kintsch y Van Dick, se identifica tres niveles de comprensión: del texto base, el nivel modelo de la situación para la integración de la información del texto (coherencia) y el nivel metacognitivo.

En el primer nivel, texto base, se trata de la interrelación de los significados del texto entre sí, de modo que constituyan un todo coherente en el que cada idea guarda una relación precisa con las demás. En este nivel se distinguen tres categorías textuales que corresponden a la microestructura, macroestructura y superestructura: 
Microestructura: Se organizan los significados de las ideas elementales del texto o unidades proposicionales, estableciéndose entre ellas una relación de coherencia.

Macroestructura: Se extrae el significado global de carácter sumativo de las ideas esenciales de los párrafos y el texto (idea central, principal, título).

Superestructura: Se refiere a la naturaleza del texto, puede ser narrativo o expositivo (descriptivo, comparativo, secuencial de proceso y causa efecto, argumentativo).

El segundo nivel, modelo de la situación, más profundo, corresponde a otra forma de integración: la integración de la información del texto con el resto de nuestros conocimientos hasta fundirse con ellos. Lo que retenemos en nuestra mente no sería el texto, sino el mundo o situación que en él se refiere.

Los niveles, texto base y modelo de la situación acentúan los dos ejes de la Psicolingüística actual: texto y contexto.

Un tercer nivel igualmente importante está representado por los procesos metacognitivos que consisten en el conocimiento de los procesos cognitivos en la actividad lectora (habilidades, estrategias y recursos para comprender lo que se lee) y los procesos de autorregulación que implican planificar, supervisar, controlar y evaluar el nivel de comprensión:

1. En primer lugar, puesto que un texto puede ser leído con diferentes propósitos, parece necesario que el lector seleccione de entre todas las metas u objetivos, uno en concreto que dirija el acercamiento al texto (planificación).

2. Asimismo, debe ser el propio lector quien efectúe la clarificación del significado del texto, detectando en primer lugar, que algo no ha sido comprendido, identificando después, el origen de esa no comprensión y, finalmente, buscando modos de resolverla (supervisión y control).

3. Finalmente, el lector debe revisar si el grado de comprensión alcanzado es aceptable o no, de acuerdo a la meta de comprensión que haya sido elaborada de antemano (evaluación).

\section{MÉTODO}

\section{Proceso de construcción de la prueba}

En la elaboración de la prueba de habilidades de pensamiento se ha tratado de operacionalizar a través de los reactivos, las definiciones conceptuales de las categorías de pensamiento comprendidas en la taxonomía de habilidades cognitivas propuestas por Reuven Feuerstein complementadas con algunas consideradas en la clasificación de Margarita Sánchez (Ordenamiento y clasificación jerárquica) y del modelo Triárquico de la Inteligencia de Robert Sternbeg (habilidad creativa verbal).

Los pasos en la construcción de la prueba fueron:

1. Definir conceptualmente las habilidades u operaciones básicas y superiores del pensamiento a evaluar. Se presentan en el Anexo A. 
2. Operacionalización de las definiciones conceptuales de cada una de las habilidades a través de indicadores expresados en reactivos seleccionados o elaborados, con preguntas frente a las cuales la persona daría una respuesta. Se presenta en el Anexo A2.

3. Estructura de la prueba: Se elaboró un conjunto de reactivos con 45 preguntas, organizados de acuerdo al nivel de complejidad desde las operaciones básicas a las operaciones lógico superiores.

4. Calificación: Se asignó un valor de 1 a las respuestas correctas, de modo que, el puntaje total de la prueba fue 45 .

\section{Fuentes de información}

Las fuentes primarias de información para la elaboración de la prueba fueron, en primer lugar, la prueba de Evaluación Dinámica de la Inteligencia de R. Feuerstein que permite analizar las operaciones y funciones cognitivas. También fueron objeto de análisis otros instrumentos y programas de desarrollo cognitivo.

Las fuentes secundarias corresponden al análisis de documentos que sustentan el marco teórico cognitivo y lingüístico e investigaciones vinculadas al tema.

\section{Proceso de Validación de la Prueba}

\section{Validez de contenido}

Se aplicó el método de Evaluación por jueces en la que participaron diez profesionales, ocho psicólogos y dos educadores con gran experiencia en evaluación educativa y en construcción de pruebas.

Los datos fueron sometidos al coeficiente $\mathrm{V}$ de Aiken, puesto que los valores son contrastados estadísticamente según el tamaño de la muestra de los jueces.

Los resultados arrojaron que casi todos los reactivos fueron considerados válidos, tanto en pertinencia como en claridad, con una probabilidad del $95 \%$. El ítem menos válido en relación a su claridad fue el de Relacion jerárquica de clases con un valor de 0.50.

La validez de contenido permitió hacer correcciones a la versión preliminar de la prueba, entre ellas tenemos:

1. Mejor ordenamiento de las respuestas correctas de la prueba en su conjunto.

2. Mejoramiento de la redacción de algunos ítems, especialmente los refranes.

3. Disminuir la cantidad de proposiciones a ordenar en el ítem 5 (Relación jerárquica de ideas).

4. Mejorar el formato de la prueba, incluyendo instrucciones específicas. 


\section{Aplicación de la Prueba Piloto}

La prueba fue aplicada a 336 sujetos, distribuidos en los grados, cuarto y quinto de secundaria e ingresantes al primero y segundo ciclo de Universidad, estatales y privados.

\section{RESULTADOS}

La evaluación psicométrica para la validación de la prueba se realizó en dos fases. En la primera fase se trabajó con la muestra de validación para analizar la confiabilidad de la prueba, utilizándose los siguientes criterios: la dificultad de los ítems (estadísticos de los elementos), la correlación ítem-test corregida para determinar la consistencia interna del instrumento, a fin de decidir qué ítems elegir y cuáles eliminar, así como la estimación del coeficiente alfa de Cronbach: si se elimina el elemento debe incrementarse de manera significativa. Como resultado de este análisis quedaron 13 ítems inválidos, de los cuales se eliminaron definitivamente cuatro.

La segunda fase consistió en incluir los nueve ítems invalidados en la muestra de normalización, previa una reevaluación inclusión o exclusión definitiva en la prueba, según los nuevos resultados.

Como parte de la evaluación psicométrica de la prueba, se dan a conocer los resultados del Análisis Factorial Exploratorio realizado en la primera fase de la validación y el Confirmatorio, después de la validación de la prueba completa en la segunda fase.

La aplicación del Análisis Factorial como uno de los métodos más significativos para evaluar la validez de constructo tuvo como uno de sus objetivos proporcionar a la prueba un fundamento científíco. La validez de constructo es definida como el grado en que una prueba mide y evalúa una característica psicológica, demostrando las construcciones teóricas que explican en cierta media el resultado encontrado (Brown, 1993; Muñiz,1994; Anastasi y Urbina, 1998).

Cuando se investiga la validez de constructo, por lo general se desea saber qué propiedad psicológica puede explicar la varianza de la prueba. El investigador se pregunta: ¿qué construcción teórica mide la prueba?, ¿qué factores o constructos dan cuenta de la varianza en el desempeño de la prueba?

La validez de constructo adquiere gran importancia en la Psicometría e investigación científica porque vincula las nociones y prácticas psicométricas con las nociones teóricas (Kerlinger, 2000). No se trata sólo de validar el instrumento sino aproximarse a la teoría que fundamenta el instrumento.

El aspecto significativo acerca de la validez de constructo es el estar focalizado en la teoría, los constructos teóricos y la investigación científica empírica que implica la prueba de relaciones hipotetizadas entre las variables. La validación de constructo requiere una teoría sustantiva para definir el constructo que va a ser medido, así como una teoría de la medición para proporcionar la medida. 


\section{Análisis psicométrico de los datos: Primera Fase}

Debido a que la muestra completa de 336 casos no tenía la suficiente variabilidad, se optó por analizar los datos mediante la técnica de los grupos extremos. La Tabla N.$^{\circ} 1$ muestra el nivel de los puntajes totales.

Tabla N. ${ }^{0}$ 1. Nivel de los Puntajes Totales.

\begin{tabular}{lcccc}
\hline \multicolumn{1}{c}{ Variable } & Frecuencia & Porcentaje & $\begin{array}{c}\text { Porcentaje } \\
\text { válido }\end{array}$ & $\begin{array}{c}\text { Porcentaje } \\
\text { acumulado }\end{array}$ \\
\hline Grupo Bajo & 119 & 35,4 & 35,4 & 35,4 \\
Grupo Medio & 99 & 29,5 & 29,5 & 64,9 \\
Grupo Alto & 118 & 35,1 & 35,1 & 100,0 \\
Total & 336 & 100 & 100 & \\
\hline
\end{tabular}

\section{Análisis de la Confiabilidad de la prueba}

El Alfa de Cronbach es sensible a dos casos: en primer lugar, el número de ítems, lo que significa que a mayor número de ítems, habrá un mayor coeficiente alfa. El otro caso compromete a la cantidad de personas: mientras más personas se usan en el cálculo, es probable que haya mayor variabilidad, y con ello un alfa más alto.

\section{Análisis de Confiabilidad de la prueba completa}

El análisis de la prueba completa (45 ítems) evidenció una consistencia interna elevada, expresada por el coeficiente Alfa de Cronbach que se presenta en la Tabla N. ${ }^{\circ} 2$.

Tabla N. ${ }^{\circ}$ 2. Coeficiente de Confiabilidad.

\begin{tabular}{cc}
\hline Alfa de Cronbach & N. $^{\circ}$ de elementos \\
\hline, 809 & 45 \\
\hline
\end{tabular}

El coeficiente Alfa de Cronbach $(, 809)$ nos permitió asegurar que la prueba en su conjunto guarda coherencia interna.

\section{Análisis de los índices de dificultad de los ítems}

Los índices de dificultad de los ítems están representados por los porcentajes de respuestas correctas a cada uno. Ello nos indica la cantidad de sujetos de la muestra que respondieron adecuadamente al ítem, aunque no nos dice en qué porcentaje respondieron correctamente los grupos alto y bajo de la muestra. Sin embargo, nos permite observar 
qué ítems contribuyeron más a la consistencia de la prueba total. La Tabla del Anexo B, Estadísticos de los elementos, muestra el índice de dificultad de los ítems con la correspondiente desviación típica, para el total de los grupos extremos.

\section{Correlaciónes entre ítem y Puntaje Total}

A través del grado de relación entre cada ítem y el puntaje total se demuestra la consistencia interna de la prueba, con el supuesto que si un ítem mide un aspecto particular de la variable, en este caso, habilidades de pensamiento, los ítems deben tener una correlación positiva con el puntaje total del test. De este modo, podemos hacer un deslinde entre los ítems que miden lo mismo de la prueba de aquellos que no, y conocer además, los ítems que contribuyen o no, a la coherencia interna de la prueba.

Este procedimiento nos permitió eliminar los ítems que obtuvieron correlaciones ítem/test negativas o bajas con valores inferiores a 0,20 (Kline,1982).

La tabla del Anexo C presenta las correlaciones entre cada uno de los ítems y puntaje total corregidas y el índice Alfa de Cronbach si se elimina el elemento.

De modo general, esta tabla permite identificar los ítems que no cumplen el criterio (los que presentan un valor de la correlación menor a 0,20 , ya sea positivo o negativo), lo cual significa que no discriminan entre los sujetos de alto y bajo desempeño en las habilidades de pensamiento. En tal sentido, 13 ítems sombreados, fueron eliminados, quedando 32 ítems válidos.

\section{Análisis de la Confiabilidad con los ítems Seleccionados}

Eliminándose los 13 ítems que no discriminan entre los buenos y deficientes alumnos lectores respecto a las habilidades de pensamiento, es decir, que no contribuyen a la coherencia interna de la prueba, los índices de confiabilidad se incrementan, a juzgar por el índice Alfa de Cronbach para la prueba en su conjunto (Tabla N. ${ }^{\circ} 3$ ).

Los estadísticos de los elementos y la correlación ítem-total corregida de los ítems seleccionados se muestran en las tablas correspondientes a los Anexos D y E, respectivamente.

Tabla N. ${ }^{\circ}$ 3. Coeficiente de Confiabilidad de la Prueba Total.

\begin{tabular}{cc}
\hline Alfa de Cronbach & N. ${ }^{\circ}$ de elementos \\
\hline, 846 & 32 \\
\hline
\end{tabular}

\section{Análisis psicométrico de los datos: Segunda Fase}

Después de un análisis de los ítems invalidados (13), se tomó la decisión de eliminar definitivamente cuatro de ellos y tratar de mejorar la redacción de nueve ítems para una revalidación, lo que conformaría una prueba de 41 ítems. 
La muestra para la revalidación de los nueve ítems fue extraída proporcionalmente de la muestra de normalización, conformando 300 sujetos.

El análisis psicométrico de los datos consideró en primer lugar la estimación de la confiabilidad de toda la prueba (41 ítems) a través del coeficiente de Cronbach, como se aprecia en la Tabla N. ${ }^{\circ} 4$ :

Tabla N. ${ }^{\circ}$ 4. Coeficiente de confiabilidad.

\begin{tabular}{cc}
\hline Alfa de Cronbach & N. $^{\circ}$ de elementos \\
\hline, 851 & 41 \\
\hline
\end{tabular}

Mediante el coeficiente Cronbach $(, 851)$ se comprueba la consistencia interna elevada de la prueba la prueba completa con 41 ítems.

\section{Análisis de los Índices de Dificultad de los Ítems}

Los índices de dificultad de los ítems que se presentan en la tabla del Anexo F muestran niveles aceptables de los promedios de porcentaje de respuestas correctas entre altos, medios y bajos.

\section{Correlaciones entre Ítem y Puntaje Total}

Las correlaciones ítem/test que se muestran en la tabla del Anexo G evidencian la consistencia interna de los 41 ítems de la prueba, presentando correlaciones positivas de 0.20 a más, lo cual significa, el grado de discriminación entre los sujetos de alto y bajo desempeño en las habilidades de pensamiento.

Los resultados también confirman que los nueve ítems eliminados en la primera fase de la validación y mejorados han pasado la prueba de validación para ser incluidos en la prueba definitiva con 41 ítems. Dichos ítems aparecen sombreados en la misma tabla del Anexo G.

\section{Análisis Factorial: Validez de constructo}

El análisis se ha realizado en base al método intraprueba a fin de identificar el grado de dimensionalidad de las variables (factores) expresadas en las operaciones del pensamiento, a través del estudio de la relación de las estructuras internas y cruzadas subyacentes $y$, complementariamente, determinar la validez teórica del instrumento. En realidad, se trata de reducir el número de dimensiones necesarias para explicar las respuestas de los sujetos.

El procedimiento seguido para la aplicación de la técnica de Análisis Factorial ha sido desarrollado, como se mencionó anteriormente, en las dos fases de la validación. En la primera, se realizó el análisis factorial exploratorio con los 32 ítems validados, en 
el que se habían eliminado 13 ítems. En la segunda fase de validación se realizó el Análisis Factorial Confirmatorio con el Programa Estadístico AMOS 16* de los 41 ítems validados que conformarían la prueba definitiva.

\section{Primera Fase del Análisis Factorial}

Con la finalidad de evaluar la viabilidad del análisis factorial se emplearon las estadísticas preliminares que se presentan en la Tabla N. ${ }^{\circ}$ 5: las medidas de adecuación muestral Kaise.Meye.Olkin (KMO) y la prueba de esfericidad de Bartlett aplicadas a los 32 ítems seleccionados.

Tabla N. ${ }^{\circ}$ 5. KMO y Prueba de Bartlett.

\begin{aligned} & \hline Medida de adecuación muestral de Kaise.Meyer. Olkin (KMO):, 816 \\ & Prueba de esfericidad de Bartlett: Ch.cuadrado aproximado: 1415,286 \\ & gl 496 \\ & Sig., 000 \\ & \hline\end{aligned}

* El Programa AMOS (Analysis of Moment Structures; Arbucide,1997) corresponde a un modelo de análisis estadístico de ecuaciones estructurales multivariantes.

La medida de adecuación muestral (KMO) contrasta si las correlaciones parciales entre las variables son suficientemente pequeñas. Permite comparar la magnitud de los coeficientes de correlación observadas con la magnitud de los coeficientes de correlación parcial. El estadístico varía entre 1 y 0.

La prueba de esfericidad de Bartlett contrasta la hipótesis nula -la matriz de correlaciones es una matriz de identidad- en cuyo caso no existirían correlaciones significativas entre las variables y el modelo factorial no sería adecuado. La prueba de esfericidad de Bartlett va a medir si la matriz de correlaciones se diferencia de la matriz de identidad: si las correlaciones son lo suficientemente altas como para continuar con el análisis.

Tanto la medida KMO como la prueba de esfericidad de Bartlett son altas, para permitir continuar con el análisis: existe una muestra adecuada para proceder al análisis factorial , y en lo que respecta a la prueba de esfericidad de Bartlett, específicamente ha permitido determinar la significación de la matriz de correlación respecto a la matriz de identidad, alcanzando un valor estadísticamente significativo.

Los puntajes satisficieron los criterios para realizar el análisis factorial.

\section{Análisis Factorial Exploratorio}

En el análisis exploratorio de los datos, por ser la muestra de validación, el criterio estadístico tiene el objetivo de explicar la mayor varianza (o propiedad relacionada) con 
el número más pequeño de factores. Es decir, saber qué factores o variables latentes no observables se encuentran detrás del desempeño de la prueba, siendo la pregunta fundamental ¿qué factores / constructos dan cuenta de la varianza en el desempeño de la prueba?

Para llegar a la solución factorial se utilizó como método de extracción, el análisis de Componentes Principales y como método de rotación, la Normalización Oblimin con Kaiser.

El método de extracción de los componentes principales asume que es posible explicar el $100 \%$ de la varianza observada y, por ello, todas las comunalidades iniciales son iguales a la unidad (que es justamente la varianza de una variable con puntuaciones típicas).

\section{Comunalidades}

La tabla del Anexo $\mathrm{H}$ contiene las comunalidades asignadas inicialmente a las variables (inicial) y las comunalidades reproducidas por la solución factorial (extracción). La comunalidad de una prueba es la proporción de su varianza que puede ser explicada por el modelo factorial obtenido o, expresada en otros términos, es su varianza de factor común. Estudiando las comunalidades de la extracción podemos valorar cuáles de las variables son peor explicadas por el modelo.

Para una comprensión del significado conceptual de las comunalidades, partimos del supuesto que la prueba está formada por variables que tienen elementos comunes que vendrían a ser los factores. La comunalidad viene a ser la proporción de varianza conjunta que comparte un grupo de ítems a través de un factor o que también puede presentar un ítem a través de uno o más factores.

A partir de la tabla del Anexo $\mathrm{H}$ podemos empezar a plantearnos si el número de factores obtenido es suficiente para explicar todas y cada una de las variables incluidas en el análisis.

En las tabla del Anexo I de los porcentajes de varianza total explicada, se ofrece un listado de autovalores de la matriz y de la varianz.covarianza , y del porcentaje de varianza que representa cada uno de ellos.

Los autovalores iniciales son entidades matemáticas por las cuales se permite calcular la proporción de la varianza explicada, es decir, expresan la cantidad de la varianza total que está explicada por cada factor y los porcentajes de varianza explicadas asociados a cada factor. Aparecen de manera descendente, desde la mayor a menor cantidad.

La Tabla N. ${ }^{\circ} 6$ resume los componentes principales que explican la varianza acumulada. Continúa la presentación de la varianza total explicada de los autovalores iniciales, la suma de las saturaciones al cuadrado de la extracción y la suma de las saturaciones al cuadrado de la rotación. 
Tabla N. ${ }^{\circ}$ 6. Varianza total explicada.

\begin{tabular}{cccccc}
\hline \multirow{2}{*}{ Componente } & $\begin{array}{c}\text { Autovalores } \\
\text { iniciales }\end{array}$ & Sumas de las saturaciones al cuadrado de la \\
extracción & $\begin{array}{c}\text { Suma de las } \\
\text { saturaciones al } \\
\text { cuadrado de la } \\
\text { rotación* }\end{array}$ \\
\cline { 2 - 6 } & \% acumulado & Total & \% de la varianza & \% acumulado & Total \\
\hline 1 & 18,229 & 5,833 & 18,229 & 18,229 & 4,029 \\
2 & 23,627 & 1,727 & 5,397 & 23,627 & 3,397 \\
3 & 28,765 & 1,644 & 5,138 & 28,765 & 3,941
\end{tabular}

Método de extracción: Análisis de Componentes principales.

* Cuando los componentes están correlacionados, las sumas de los cuadrados de las saturaciones no se pueden añadir para obtener una varianza total.

La tabla del Anexo J muestra la matriz de Componentes o Factores. Se identifican los ítems de los factores que presentan la mayor carga factorial.

En la tabla del Anexo K se puede apreciar la matriz de configuración: mediante el método de extracción de análisis de componentes principales y el método de rotación Oblicua de tipo Oblimin se verificó la existencia de tres factores que explican el porcentaje de la varianza total.

La tabla del Anexo L muestra la matriz de estructura con los ítems agrupados en cada factor o componente, con los dos métodos expuestos anteriormente.

La Tabla N. ${ }^{\circ} 7$ presenta el valor de las correlaciones que existen entre los tres factores o componentes.

Tabla N. ${ }^{0}$ 7. Matriz de correlaciones entre componentes.

\begin{tabular}{cccc}
\hline Componente & 1 & 2 & 3 \\
\hline 1 & 1,000 & & \\
2 &, 252 & & \\
3 &,- 270 &,- 270 & 1,000 \\
\hline
\end{tabular}

Método de extracción: Análisis de componentes principales.

Metodo de rotación: Normalización Oblimin con Kaiser.

Los tres factores identificados se constituyen de la siguientes forma: 
Factor I, que incluye ítems, principalmente de Inferencia lógica deductiva e inductiva independientemente de su complejidad, y planteamiento de hipótesis (predicción).

Factor II, comprende ítems relacionados con abstracciones, generalizaciones e ideas globales (capacidad sumativa).

Factor III, constituido por ítems relacionados principalmente con el razonamiento analógico.

\section{Segunda Fase del Análisis Factorial}

Tuvo como objetivo identificar si los 41 ítems entre los cuales se habían incluido los nueve ítems revalidados, tenían la misma estructura factorial de los 32 ítems de la primera fase de validación y dar lugar al análisis factorial confirmatorio. Esta fase se desarrolló aplicando el modelo de análisis estadístico multivariante del Programa AMOS 16 como ya se ha mencionado.

Los resultados de este análisis demostraron ser favorables a la estructura de los tres factores. En el Anexo M se presentan las tablas de confiabilidad de los tres factores identificados a través del Análisis Exploratorio en la primera muestra de validación.

\section{DISCUSIÓN}

El presente estudio psicométrico cuyos resultados se han dado a conocer, tuvo como objetivo validar un instrumento elaborado para la evaluación cuantitativa, así como cualitativa, en términos de un análisis dinámico y funcional de las habilidades de pensamiento a través de la lectura en estudiantes de cuarto y quinto grado de secundaría y primer año de la Universidad.

El desarrollo de las habilidades de pensamiento representa actualmente uno de los temas de interés tanto a nivel científico como educativo. Los nuevos paradigmas educativos centran su atención en modelos de enseñar a pensar, ya sea en forma directa o a través de las áreas curriculares para que los alumnos desarrollen su potencial de aprendizaje y aprendan a aprender de forma eficaz.

Los resultados analizados han permitido fundamentar de forma científica y válida a nivel teórico y metodológico un instrumento elaborado con fines de diagnóstico que pueda ser utilizado en un modelo integrado de investigación y desarrollo aplicable a la construcción, implantación y evaluación de proyectos para enseñanza y transferencia de habilidades de pensamiento, como lo propone Feuerstein, cuya Teoría de Modificabilidad Cognitiva Estructural constituye la base del Programa de Enriquecimiento Instrumental (PEI) y la Evaluación Dinámica del Potencial de Aprendizaje (LPAD) $(1991,1993$, 1997), en niños, jóvenes y adultos con necesidades educativas y, M. Sánchez (2002), en el marco de la teoría basada en procesos como área curricular en ambientes de educación regular para mejorar el potencial de aprendizaje.

El proceso de validación de la prueba se inició con un análisis lógico y sistemático del contenido de los ítems de la prueba respecto a su pertinencia y claridad; es decir, 
si los ítems representaban realmente las definiciones operacionales de los constructos propuestos por los autores de la prueba, en este caso, las habilidades de pensamiento. Participaron en calidad de jueces, diez profesionales, ocho psicólogos y dos educadores de amplia experiencia en evaluación.

Es importante mencionar que la prueba contiene un conjunto de enunciados, unos corresponden a planteamientos de un problema y otros a textos, seguidos de preguntas en términos de la demanda cognitiva (habilidades de pensamientos) que plantea su solución) vinculada a las operaciones básicas y superiores del pensamiento de la taxonomía propuesta por Feuerstein: clasificación, análisis-síntesis, inferencia lógica, razonamiento analógico, razonamiento hipotético, razonamiento transitivo, razonamiento silogístico, complementada con la habilidad creativa verbal (razonamiento divergente) en la terminología de Sternberg y clasificación jerárquica de la taxonomía de Sánchez.

Los resultados demostraron en general, validez de contenido de los ítems (con una probabilidad de $95 \%$ ) con sugerencias de mejoras en el aspecto formal.

El análisis psicométrico de los datos se realizó en dos fases y en cada una de ellas se aplicaron los estadísticos de confiabilidad (análisis de los índices de dificultad de los ítems, coeficiente alfa de Cronbach para la prueba completa y las correlaciones entre ítem y Puntaje total. En la primera fase se aplicaron dichos estadísticos a 45 ítems de la versión original, quedando 32 ítems válidos; en la segunda, se procedió al procesamiento estadístico con 41 ítems, de los cuales nueve fueron revisados y corregidos para ser reevaluados, eliminándose definitivamente cuatro.

Los análisis de los coeficientes Alfa de Cronbach tanto de la prueba en su conjunto (0.85) como de cada uno de los 41 ítems validados (entre 0,83 y 0,85 ) si se elimina el elemento, demostraron que la prueba de habilidades es una medida confiable en términos de consistencia interna y estabilidad. Se aprecia asimismo, que la correlaciones ítem/test corregidas son aceptables (entre ,21 y ,50) en los 41 ítems de la prueba.

La validez de constructo es la principal de los tipos de validez, en tanto es el concepto unificador que integra las consideraciones de validez de contenido y de criterio en un marco común para probar hipótesis acerca de las relaciones teóricamente relevantes (Messick, 1980).

En el presente trabajo se realizó el Análisis Exploratorio en la primera fase de validación con los treinta y dos ítems validados y el Análisis Factorial Confirmatorio en la segunda fase, después de la validación de la prueba completa con 41 ítems, con la aplicación del Programa Amos 16.

En la primera fase, las estadísticas preliminares aplicadas a la matriz de correlaciones entre las variables satisficieron los criterios para proceder al Análisis Factorial: la medida de adecuación muestral Kaiser-Mayer-Olkin (KMO) demostró que las correlaciones parciales eran los suficientemente pequeñas con un valor de .81; la prueba de esfericidad de Bartlett (Bondad de Ajuste) rechazó la hipótesis nula -la matriz de correlaciones es una matriz de identidad- en cuyo caso no existirían correlaciones significativas entre las variables y el modelo factorial no sería adecuado. El valor obtenido fue también estadísticamente significativo. 
El Análisis Factorial Exploratorio, mediante un conjunto de métodos multivariados de interdependencia tuvo como objetivo principal identificar la estructura de factores subyacentes a un conjunto amplio de datos. Para llegar a la solución factorial se utilizó como método de extracción el Análisis de los Componentes Principales (factores) y como método de rotación, la rotación Oblimin con Kaiser, debido a que se consideró necesario verificar que los factores sean correlacionados.

Se identificaron tres factores (dimensiones) que explican la variabilidad del desempeño de la prueba:

Factor 1. Inferencia deductiva e inductiva y planteamiento de hipótesis (predicción), silogismos, independientemente de su complejidad, en dieciocho ítems (1, 2, 3, 21, 27, $29,30,31,32,33,34,35,36,37,38,39,40,41)$. Este factor subyace no sólo en los ítems reconocidos como inferencia lógica propiamente dicha, sino en aquellos vinculados a los constructos creatividad verbal y razonamiento transitivo, por la demanda cognitiva de la pregunta de carácter inferencial.

La inferencia lógica constituye un factor clave en la construcción del significado. Goodman (1982), en relación a este proceso, expresa: "La inferencia es un medio poderoso por el cual las personas complementan la información disponible, utilizando el conocimiento conceptual, lingüístico y los esquemas que ya poseen. Los lectores emplean estrategias de inferencia para encontrar lo que no está explícito en el texto. Pero también infieren cosas que se harán explícitas más adelante”.

Se ha demostrado que la inferencia es una operación del pensamiento que explica las diferencias entre buenos y deficientes lectores en alumnos de segundo a quinto de secundaria (Tapia,V., Luna, J., 2008). Asimismo, en un estudio comparativo sobre comprensión lectora en estudiantes de tercer grado de Secundaria, considerando el tipo de centro educativo, Delgado, A., Escurra, M. Pequeña, J. y otros (2009) encontraron diferencias significativas en el nivel de comprensión lectora inferencial a favor de los alumnos de centros educativos no estatales.

Factor 2. Abstracciones, generalizaciones, ideas globales, en catorce ítems $(4,5,7,8$, $10,12,13,14,15,16,17,18,19,20)$ Este factor subyace en ítems relacionados con la capacidad sumativa e incluye constructos de clasificación, clasificación jerárquica, conceptualización y síntesis. Está relacionado con preguntas sobre el significado de una palabra, colocar un título, extraer la idea principal, resumir.

También se ha comprobado que los deficientes lectores presentan dificultades de abstracción, vinculada a una función deficiente en el proceso de elaboración: conducta episódica de la realidad. Los estudiantes poco estratégicos y los deficientes lectores procesan la información a trozos y asistemáticamente, focalizando la atención primordialmente en las palabras mismas más que en su significado y en el significado que cada frase tiene en sí misma sin relacionarlo con el contexto de los párrafos o el contenido global de todo el texto (Garner 1980, citado por Buron, 1999).

La importancia de esta capacidad de abstracción es reconocida como una de las competencias generales de mayor importancia en los perfiles profesionales universitarios en Latinoamérica (Informe Final del Proyecto Tuning, 2007). 
Aún siendo independientes, los factores 1 y 2 se relacionan en la medida que para realizar inferencias, se debe tener una percepción global del texto.

Factor 3. Razonamiento analógico, constituido por nueve ítems $(6,9,11,22,23,24$, $25,26,28)$. Este factor constituye un constructo subyacente en ítems en los que la demanda cognitiva de la pregunta requiere un proceso de establecimiento de relaciones de diferencia y semejanza que se establecen entre términos o proposiciones, relaciones que pueden ser concretas o abstractas. La solución a la pregunta del ítem se determina por la deducción de la semejanza.

Esta categoría de razonamiento está representada en la prueba principalmente a través de los ítems que comprenden refranes y pensamiento metafórico (habilidad creativa verbal).

Con la identificación de los tres factores que subyacen en las diferentes operaciones del pensamiento se ha determinado la validez de constructo de la prueba, a nivel teórico, respondiendo a la pregunta sobre las dimensiones, factores o constructos que explican la varianza del desempeño de la prueba. Es decir, las nueve variables (habilidades de pensamiento) en las que se agrupan los 41 ítems de la prueba, se han reducido a tres dimensiones que explican las diferencias individuales en los puntajes de la prueba.

No obstante, el propósito principal del estudio es poner al alcance de los educadores, psicólogos y otros profesionales vinculados con el tema, un instrumento con fundamento teórico y metodológico, es importante advertir que es sólo el inicio de un proceso de análisis e interpretación de los resultados para un diagnóstico individual o grupal del nivel alcanzado por los estudiantes que terminan la secundaria y están cursando el primer año de educación superior universitaria. Para un análisis cualitativo y funcional de los resultados, de acuerdo al enfoque que proponemos, se requiere tener en cuenta los diferentes aspectos de los fundamentos teóricos analizados, tales como el análisis de las funciones involucradas y los niveles del procesamiento de textos presentados en la primera parte del estudio.

\section{CONCLUSIONES}

1. La prueba de habilidades de pensamiento ha demostrado tener confiabilidad, tanto en su totalidad como en los diferentes ítems que la conforman, por consistencia interna

2. Los índices de dificultad a través de promedio de respuestas correctas a cada ítem son adecuados.

3. La Prueba de Habilidades de Pensamiento tiene validez de constructo, evaluado a través del Análisis Factorial, lo que significa que se ha validado la teoría de que fundamenta el instrumento.

4. Tres factores o constructos dan cuenta de la variabilidad del desempeño de la prueba: el pensamiento inferencial, la capacidad de generalizar (abstraer); y el razonamiento analógico.

5. Cada uno de los factores identificados y sus respectivos ítems presentan consistencia interna estimada por los índices de confiabilidad. 


\section{REFERENCIAS BIBLIOGRÁFICAS}

1. Alarcón, N. R. (1991). Métodos y diseños de investigación del comportamiento. Universidad Cayetano Heredia.

2. Beneitone, P.; Esquetin , C.; Gonzáles, J.; Marty, M.; Siufi, G.; Wagenaar, R. (editores) (2007). Informe final del proyecto Tuning América Latina. Universidad de Deusto Bilbao.

3. Buron, J. (1999). Enseñar a aprender: Introducción a la metacognición. Ediciones Mensajero. España.

4. Cassany, D. (2005). El significado de la comprensión crítica. En Lectura y vida. Revista Latinoamericana de lectura, año 26, setiembre.

5. Delgado, A.; Escurra, M. Pequeña, J y otros (2009). Comparación de la comprensión lectora en alumnos de tercer año de secundaria de centros educativos estatales y no estatales de Lima Metropolitana. En: PERSONA Revista de la Facultad de Psicología. Universidad de Lima, N. ${ }^{\circ}$ 12, ener.diciembre, ISSN 156.6139. Lima, Perú.

6. Ferrando, P.J.; Angulario-Carrasco, C. ( 2010). El Análisis factorial como técnica de investigación en Psicología. Centro de Investigación para la evaluación y medida de la conducta. Universidad Roviray Virgili. Papeles del Psicólogo Vol. 31/21.

7. Feuerstein, R. (1997). Teoría de la modificabilidad cognitiva estructural. En: Martínez, J. M.; Tebar, L.; Rivera, J. A (Dirección) ¿Es modificable la inteligencia? Edit. Bruño.

8. Feuerstein, R. y Feuerstein S. (1991). Mediated Learning Experience (MLE): Theoretical, psychosocial and learning implications. En: Feuerstein, R.; Klein, P. y Tannenbaum, A. (editores) International Center for Enhancement of Learning Potential (ICELP).

9. Feuerstein, R.; Hoffman, M. (1991). Programa de Enriquecimiento Instrumental. Apoyo Didáctico 1 y 2. Instituto Superior Pío XII España.

10. Feuerstein, R. y Ben Shachar, N. (1993). La evaluación dinámica del potencial de Aprendizaje. Hadassah Wizo Canada Research Institute.

11. Gardner, H. (1993). La mente no escolarizada: Cómo piensan los niños y cómo deberían enseñar las escuelas. Edit. Paidos.

12. Johnson A. (2003). El desarrollo de las habilidades del pensamiento. Aplicación y planificación para cada disciplina. Edit. Troquel.

13. Kerlinger, F. (1988). Análisis Factorial. En: Investigación del comportamiento. McGraw Hill.

14. Martínez, J. M. (1998). Análisis y modificación del acto mental. La metodología de la mediación en el PEI. Edit. Bruño.

15. Nunnally, J. y Bernstein, J. (1995). Análisis factorial. En: Teoría psicométrica. McGraw Hill.

16. Pérez J. A.; Chacón, S.; Moreno, R. (2000). Validez de constructo: El uso de análisis factorial exploratorio, confirmatorio para obtener evidencias de validez. Universidad de Sevilla Psicothema,Vol. 12, N. ${ }^{\circ}$ 12, pp. 442-495. 
17. Pérez, E; Medrano, L. (2010). Análisis factorial exploratorio: bases conceptuales y metodológicas. Revista Argentina de Ciencias del Comportamiento (RACC).

18. Ruiz, M.; Pardo, A San Martín, R. (2010). Modelos de ecuaciones estructurales. Facultad de Psicología. Universidad de Madrid. Papeles del Psicólogo, Vol. 31(1).

19. Sánchez, E. (1998). El lenguaje escrito: adquisición e intervención educativa. En: Beltrán, J. y Genovard, C. (Edit.) Psicología de la instrucción I: Variables y procesos básicos. Edit Síntesis, Madrid.

20. Sternberg, R. y Spea.Swerling, L. (1999). Enseñar a pensar. Aula XXI. Edit. Santillana.

21. Sánchez, M, (2002). La investigación sobre el desarrollo y la enseñanza de las habilidades del pensamiento. En: Revista Electrónica de Investigación Educativa, Vol. 4, N. ${ }^{\circ} 1$.

22. Tapia, V. (1998). Aportes de Reuven Feuerstein a la Psicología y la Educación. En: Seminario taller y didáctica creativa. Universidad Ricardo Palma.

23. Tapia, V. y Escurra L.M. (2002). Validez de constructo de la batería Woodcock de proficiencia en el idioma. Revista de Investigación en Psicología N. o 9, Vol. 1.

24. Tapia, V. (2005). La Modificabilidad Cognitiva Estructural. En: Conferencia Congreso Internacional de Psicología. Facultad de Psicología,Universidad Nacional Mayor de San Marcos.

25. Tapia, V.; Luna, J. (2008). Procesos cognitivos y desempeño lector. Facultad de Psicología de la UNMSM. En: Revista de Investigación en Psicología. N. ${ }^{\circ}$ 11, Vol. 1. 


\section{ANEXOS \\ ANEXO A1 \\ DEFINICIÓN CONCEPTUAL DE LAS HABILIDADES DE PENSAMIENTO}

\section{Operaciones básicas}

Identificación. Conocimiento de una realidad por sus características globales. Permite concluir si un objeto, realidad o hecho pertenece o no a un concepto.

Comparación. Habilidad que permite apreciar las características semejantes y diferentes que se observan en diversos objetos, hechos, fenómenos o procesos y en trozos de información en relación a atributos específicos.

Clasificación. Puede considerarse como una extensión de la comparación mediante la cual se agrupan elementos en categorías definitorias sobre la base de sus atributos.

La clasificación está basada en comparaciones exitosas de diferenciación y discriminación. Los conjuntos están formados sobre las bases de las semejanzas compartidas por objetos o eventos, mientras los subconjuntos están formados sobre las bases de las diferencias entre los miembros.

Ordenamiento y clasificación jerárquica. Significa estructurar las ideas generales y específicas en una secuencia coherente que permita establecer la relación entre las partes del texto y el sentido global del mismo. Es una manera más compleja de categorización.

Análisis-síntesis. El análisis significa descomponer un todo en sus elementos constitutivos y relacionarlos para extraer información. La diferenciación, la división de un todo en sus partes, la integración o la síntesis en un todo dado, son requisitos para la adaptación de una persona al medio.

Inferencia lógica. Esta actividad constituye un proceso clave en la construcción del significado. Es entendida como la capacidad de obtener información o establecer conclusiones que no están expresadas de manera explícita en el texto o en la situación comunicativa.

La inferencia inductiva consiste en extraer conclusiones a partir de trozos de información específicos inestructurados y estructurados.

La inferencia deductiva consiste en extraer conclusiones basadas en un principio o generalización explícita o asumida.

\section{Habilidades de razonamiento lógico verbal superiores}

Razonamiento hipotético. Capacidad mental de realizar inferencias y predicción de hechos a partir de los ya conocidos y de las leyes que los relacionan. Las conclusiones a las que se llegan son por inducción y deducción. 
Razonamiento analógico. Capacidad para establecer relaciones de semejanza entre diferentes términos o proposiciones, relaciones que pueden ser concretas y abstractas. En su forma de presentación más simple es "la operación por la cual dados tres términos de una proposición, se determina el cuarto por la deducción de la semejanza" ejemplo: madre / padre $=$ como abuela / abuelo.

Razonamiento transitivo. Consiste en ordenar, comparar y describir las relaciones que existen entre conjuntos ordenados, de modo que se llegue a una conclusión. La diferencia entre los miembros de un conjunto se describe en términos de "mayor qué", "igual a" "menor que".

Es posible expresar las relaciones mediante los conceptos: fuerza, tamaño, edad, temperatura, rapidez, anchura, color, peso, área, altura y otros.

Silogismos. Se refieren a la lógica proposicional formal. Su práctica intenta hacernos más críticos al analizar proposiciones y enunciados para comprobar su veracidad.

Pensamiento Crítico (literacidad crítica en el contexto de la lectura). En la tradición humanístic.liberal que aún predomina, el pensamiento crítico refiere a un grado superior de comprensión, definido como el proceso de evaluar la autenticidad y la validez de la información y de formular una opinión sobre ella. El pensamiento crítico se propone analizar y evaluar la estructura y consistencia de los razonamientos, particularmente opiniones o afirmaciones que la gente acepta como verdadera en el contexto de la vida cotidiana.

Este proceso abarca aspectos diversos, tales como la capacidad de hacer inferencias, distinguir los hechos de las opiniones y la verdad de la fantasía, detectar el sesgo o el prejuicio, identificar la trama, analizar elementos literarios, descubrir la intención del autor o fomentar la interpretación personal (Cassany, 2005).

Habilidad creativa verbal. Denominada también pensamiento original, imaginación constructiva, pensamiento divergente. Es un proceso cognitivo que implica generar ideas o conceptos, integrarlos o ver cosas de manera nueva u original. Genera un producto, un desempeño o un paradigma nuevo o mejorado, en general, un resultado final que representa el criterio para valorar la creatividad.

El pensamiento creativo incluye una dialéctica incesante entre integración y expansión, convergencia y divergencia (Harper, 1984). 


\section{ANEXO A2 \\ DEFINICIONES OPERACIONALES DE LAS HABILIDADES DE PENSAMIENTO}

\begin{tabular}{lc}
\hline Habilidades de pensamiento & Definiciones operacionales \\
\hline & Reconoce información explícita sobre ideas, objetos, hechos,
\end{tabular}

Identificación

Comparación

Clasificación

Clasificación jerárquica

Síntesis

Inferencia

Razonamiento analógico

Razonamiento hipotético

Razonamiento silogístico

Razonamiento transitivo

Razonamiento crítico

Pensamiento creativo verbal
Reconoce información explícita sobre ideas, objetos, hechos, situaciones, características.

Establece relaciones de semejanza y diferencia, considerando uno o más criterios.

Define significado de palabras, identifica categorías de atributos.

Se ordenan proposiciones de acuerdo a un orden lógico y relaciones de mayor a menor generalidad.

Identifica el título de un texto, el tema de un texto, las ideas principales y secundarias de un párrafo o texto.

Extrae información implícita de un texto o párrafo, elaborando deducciones y creando nueva información a partir de datos conocidos.

Establece relaciones de semejanza entre términos de una proposición usando el argumento inductivo.

Infiere y predice hechos a partir de los ya conocidos.

Establece nuevas relaciones partir de otras utilizando el razonamiento lógico.

Se ordenan, comparan y describen hechos características y situaciones de modo que se llegue a una conclusión.

Descubre la intención del autor, distingue los hechos de las opiniones, y la verdad de la fantasía.

Establece nuevas relaciones sobre lo que ya se conoce o percibe cosas de manera original. 
ANEXO B

ESTADÍSTICOS DE LOS ELEMENTOS (ÍNDICE DE DIFICULTAD)

\begin{tabular}{|c|c|c|c|}
\hline Ítem & Dificultad & Desviación típica & N. ${ }^{\circ}$ \\
\hline Ítem 1 & ,92 &, 265 & 237 \\
\hline Ítem 2 &, 57 & ,497 & 237 \\
\hline Ítem 3 &, 78 & ,412 & 237 \\
\hline Ítem 4 &, 58 & ,494 & 237 \\
\hline Ítem 5 &, 30 &, 459 & 237 \\
\hline Ítem 6 &, 31 &, 464 & 237 \\
\hline Ítem 7 &, 32 & ,468 & 237 \\
\hline Ítem 8 &, 43 & ,497 & 237 \\
\hline Ítem 9 &, 40 & ,491 & 237 \\
\hline Ítem 10 & ,78 & ,418 & 237 \\
\hline Ítem 11 &, 24 & ,428 & 237 \\
\hline Ítem 12 &, 56 & ,498 & 237 \\
\hline Ítem 13 &, 40 & ,490 & 237 \\
\hline Ítem 14 &, 17 & ,379 & 237 \\
\hline Ítem 15 & ,34 & ,475 & 237 \\
\hline Ítem 16 & ,48 &, 501 & 237 \\
\hline Ítem 17 & ,49 &, 501 & 237 \\
\hline Ítem 18 &, 57 & ,497 & 237 \\
\hline Ítem 19 &, 40 & ,490 & 237 \\
\hline Ítem 20 &, 14 &, 351 & 237 \\
\hline Ítem 21 &, 26 &, 440 & 237 \\
\hline Ítem 22 & ,20 & ,403 & 237 \\
\hline Ítem 23 & ,46 & ,499 & 237 \\
\hline Ítem 24 &, 64 & ,482 & 237 \\
\hline Ítem 25 & ,78 & ,412 & 237 \\
\hline Ítem 26 & ,66 & ,475 & 237 \\
\hline Ítem 27 &, 81 & ,396 & 237 \\
\hline Ítem 28 &, 73 & ,443 & 237 \\
\hline Ítem 29 &, 74 & ,440 & 237 \\
\hline Ítem 30 &, 37 & ,483 & 237 \\
\hline Ítem 31 &, 53 &, 500 & 237 \\
\hline Ítem 32 &, 17 & ,379 & 237 \\
\hline Ítem 33 & ,41 & ,494 & 237 \\
\hline Ítem 34 &, 29 &, 453 & 237 \\
\hline Ítem 35 & ,61 & ,488 & 237 \\
\hline Ítem 36 &, 59 &, 492 & 237 \\
\hline Ítem 37 &, 70 & ,457 & 237 \\
\hline Ítem 38 &, 56 & ,498 & 237 \\
\hline Ítem 39 &, 47 &, 500 & 237 \\
\hline Ítem 40 &, 28 &, 451 & 237 \\
\hline Ítem 41 &, 19 & ,390 & 237 \\
\hline Ítem 42 &, 42 & ,495 & 237 \\
\hline Ítem 43 &, 21 & ,406 & 237 \\
\hline Ítem 44 &, 55 & ,499 & 237 \\
\hline Ítem 45 & ,41 & ,494 & 237 \\
\hline
\end{tabular}


ANEXO C

ESTADÍSTICOS TOTA.ELEMENTO

\begin{tabular}{|c|c|c|c|c|}
\hline Ítem & $\begin{array}{l}\text { Media de la escala si se } \\
\text { elimina el elemento }\end{array}$ & $\begin{array}{l}\text { Varianza de la escala si } \\
\text { se elimina el elemento }\end{array}$ & $\begin{array}{l}\text { Correlación element.total } \\
\text { corregida }\end{array}$ & $\begin{array}{l}\text { Alfa de Cronbach si se } \\
\text { elimina el elemento }\end{array}$ \\
\hline Ítem 1 & 20,31 & 44,748 & 210 & ,808 \\
\hline Ítem 2 & 20,67 & 43,257 & 304 & 805 \\
\hline Ítem 3 & 20,45 & 44,664 &, 120 & 810 \\
\hline Ítem 4 & 20,65 & 43,568 & 258 & 806 \\
\hline Ítem 5 & 20,93 & 44,012 & ,208 & 807 \\
\hline Ítem 6 & 20,92 & 45,684 &,- 065 &, 815 \\
\hline Ítem 7 & 20,91 & 44,327 & 211 & 809 \\
\hline Ítem 8 & 20,80 & 44,637 & ,092 & 811 \\
\hline Ítem 9 & 20,83 & 43,692 &, 240 & ,807 \\
\hline Îtem 10 & 20,46 & 43,046 & ,415 & 802 \\
\hline Ítem 11 & 20,99 & 44,042 & 223 & 807 \\
\hline Ítem 12 & 20,68 & 42,458 &, 430 & 800 \\
\hline Ítem 13 & 20,84 & 42,409 &, 445 & 800 \\
\hline Ítem 14 & 21,06 & 45,276 & 014 & 812 \\
\hline Ítem 15 & 20,89 & 44,937 & 052 &, 812 \\
\hline Ítem 16 & 20,76 & 42,703 & ,388 & 802 \\
\hline Îtem 17 & 20,74 & 42,412 & 434 & 800 \\
\hline Îtem 18 & 20,67 & 42,969 & 350 & 803 \\
\hline Ítem 19 & 20,84 & 43,028 & ,346 & 803 \\
\hline Îtem 20 & 21,09 & 45,988 &,- 130 & 815 \\
\hline Ítem 21 & 20,97 & 44,775 & 089 & 811 \\
\hline Ítem 22 & 21,03 & 43,851 & 277 & 806 \\
\hline Ítem 23 & 20,77 & 42,897 &, 359 & ,803 \\
\hline Ítem 24 & 20,59 & 44,598 &, 103 & 811 \\
\hline Ítem 25 & 20,45 & 42,901 & ,449 & 801 \\
\hline Ítem 26 & 20,57 & 42,805 & ,396 & 802 \\
\hline Ítem 27 & 20,43 & 43,229 & 404 & 802 \\
\hline Ítem 28 & 20,50 & 43,276 & 347 & 803 \\
\hline Îtem 29 & 20,49 & 42,709 & 450 & 800 \\
\hline Îtem 30 & 20,86 & 43,872 & 217 & 807 \\
\hline Îtem 31 & 20,70 & 43,540 & 258 & 806 \\
\hline Ítem 32 & 21,06 & 44,022 & 264 & 806 \\
\hline Îtem 33 & 20,82 & 44,683 & 086 & ,811 \\
\hline Ítem 34 & 20,95 & 44,891 & 065 & 812 \\
\hline Ítem 35 & 20,62 & 42,974 & ,356 &, 803 \\
\hline Ítem 36 & 20,64 & 42,164 & ,483 & 799 \\
\hline Îtem 37 & 20,53 & 42,454 & 475 & ,799 \\
\hline Ítem 38 & 20,68 & 41,881 &, 522 & ,797 \\
\hline Îtem 39 & 20,76 & 42,683 & ,391 & 802 \\
\hline Ítem 40 & 20,95 & 44,294 &, 166 & 809 \\
\hline Ítem 41 & 21,05 & 45,341 & ,000 & 812 \\
\hline Îtem 42 & 20,81 & 43,595 & 253 & ,806 \\
\hline Ítem 43 & 21,03 & 44,576 &, 139 & 809 \\
\hline Îtem 44 & 20,68 & 42,607 & ,405 & 801 \\
\hline Ítem 45 & 20,82 & 43,200 & ,316 & 804 \\
\hline
\end{tabular}

ESTADÍSTICOS DE LA ESCALA

\begin{tabular}{cccc}
\hline Media & Varianza & Desviación típica & N. $^{\circ}$ de elementos \\
\hline 21,23 & 45,493 & 6,745 & 45 \\
\hline
\end{tabular}

Se eliminaron los ítems resaltados por obtener correlaciones Íte.test negativas o bajas con valores inferiores a 0.20 (Kline 1982). 
ANEXO D

ANÁLISIS DE FIABILIDAD DE LOS ÍTEMS SELECCIONADOS

ESTADÍSTICOS DE FIABILIDAD

\begin{tabular}{cc}
\hline Alfa de Cronbach & N. ${ }^{\circ}$ de elementos \\
\hline, 846 & 32 \\
\hline
\end{tabular}

ESTADÍSTICOS DE LOS ELEMENTOS

\begin{tabular}{cccc}
\hline Ítem & Media & Desviación típica & N. \\
Ítem 1 &, 92 &, 265 & 237 \\
Ítem 2 &, 57 &, 497 & 237 \\
Ítem 4 &, 58 &, 494 & 237 \\
Ítem 5 &, 30 &, 459 & 237 \\
Ítem 7 &, 32 &, 468 & 237 \\
Ítem 9 &, 40 &, 491 & 237 \\
Ítem 10 &, 78 &, 418 & 237 \\
Ítem 11 &, 24 &, 428 & 237 \\
Ítem 12 &, 56 &, 498 & 237 \\
Ítem 13 &, 40 &, 490 & 237 \\
Ítem 16 &, 48 &, 501 & 237 \\
Ítem 17 &, 49 &, 501 & 237 \\
Ítem 18 &, 57 &, 497 & 237 \\
Ítem 19 &, 40 &, 490 & 237 \\
Ítem 22 &, 20 &, 403 & 237 \\
Ítem 23 &, 46 &, 499 & 237 \\
Ítem 25 &, 78 &, 412 & 237 \\
Ítem 26 &, 66 &, 475 & 237 \\
Ítem 27 &, 81 &, 396 & 237 \\
Ítem 28 &, 73 &, 443 & 237 \\
Ítem 29 &, 74 &, 440 & 237 \\
Ítem 30 &, 37 &, 483 & 237 \\
Ítem 31 &, 53 &, 500 & 237 \\
Ítem 32 &, 17 &, 379 & 237 \\
Ítem 35 &, 61 &, 488 & 237 \\
Ítem 36 &, 59 &, 492 & 237 \\
Ítem 37 &, 70 &, 457 & 237 \\
Ítem 38 &, 56 &, 498 & 237 \\
Ítem 39 &, 47 &, 500 & 237 \\
Ítem 42 &, 42 &, 495 & 237 \\
Ítem 44 &, 55 &, 499 & 237 \\
Ítem 45 &, 41 &, 494 & 237 \\
\hline
\end{tabular}


ANEXO E

ESTADÍSTICOS TOTA.ELEMENTO

\begin{tabular}{|c|c|c|c|c|}
\hline Ítem & $\begin{array}{l}\text { Media de la escala } \\
\text { si se elimina el } \\
\text { elemento }\end{array}$ & $\begin{array}{l}\text { Varianza de la escala } \\
\text { si se elimina el } \\
\text { elemento }\end{array}$ & $\begin{array}{l}\text { Correlación } \\
\text { element.total } \\
\text { corregida }\end{array}$ & $\begin{array}{l}\text { Alfa de Cronbach } \\
\text { si se elimina el } \\
\text { elemento }\end{array}$ \\
\hline Ítem 1 & 15,84 & 37,819 & ,208 & 845 \\
\hline Ítem 2 & 16,20 & 36,561 & 293 &, 843 \\
\hline Ítem 4 & 16,19 & 36,796 & 255 &, 844 \\
\hline Ítem 5 & 16,47 & 37,072 & 230 &, 845 \\
\hline Ítem 7 & 16,45 & 37,248 & 203 & ,846 \\
\hline Ítem 9 & 16,37 & 36,776 & 261 &, 844 \\
\hline Ítem 10 & 15,99 & 36,339 & ,408 &, 840 \\
\hline Ítem 11 & 16,53 & 37,199 & 227 &, 845 \\
\hline Ítem 12 & 16,21 & 35,650 & ,449 & ,838 \\
\hline Ítem 13 & 16,37 & 35,548 & ,476 & ,838 \\
\hline Ítem 16 & 16,29 & 35,919 & ,400 &, 840 \\
\hline Ítem 17 & 16,28 & 35,736 & ,431 & ,839 \\
\hline Ítem 18 & 16,20 & 36,222 & 351 &, 841 \\
\hline Ítem 19 & 16,37 & 36,311 & 342 &, 842 \\
\hline Ítem 22 & 16,57 & 36,967 & 294 & ,843 \\
\hline Ítem 23 & 16,31 & 36,273 & 340 &, 842 \\
\hline Ítem 25 & 15,98 & 36,279 & ,427 &, 840 \\
\hline Ítem 26 & 16,11 & 36,259 & 364 & ,841 \\
\hline Ítem 27 & 15,96 & 36,418 & ,417 &, 840 \\
\hline Ítem 28 & 16,03 & 36,406 & 368 & ,841 \\
\hline Ítem 29 & 16,03 & 35,953 & ,459 & ,838 \\
\hline Ítem 30 & 16,40 & 36,868 & ,250 & ,844 \\
\hline Ítem 31 & 16,24 & 36,455 & 309 & ,843 \\
\hline Ítem 32 & 16,59 & 37,259 & 252 &, 844 \\
\hline Ítem 35 & 16,16 & 36,192 & ,364 & ,841 \\
\hline Ítem 36 & 16,17 & 35,398 & ,500 & ,837 \\
\hline Ítem 37 & 16,06 & 35,670 & ,493 & ,837 \\
\hline Ítem 38 & 16,21 & 35,108 &, 545 &, 835 \\
\hline Ítem 39 & 16,30 & 36,031 & ,381 & ,841 \\
\hline Ítem 42 & 16,35 & 36,973 & ,224 & ,845 \\
\hline Ítem 44 & 16,22 & 35,757 & ,430 & ,839 \\
\hline Ítem 45 & 16,35 & 36,577 & ,293 &, 843 \\
\hline
\end{tabular}

\section{ESTADÍSTICOS DE LA ESCALA}

\begin{tabular}{cccc}
\hline Media & Varianza & Desviación típica & N. ${ }^{\circ}$ de elementos \\
\hline 16,77 & 38,569 & 6,210 & 32 \\
\hline
\end{tabular}




\section{ANEXO F}

\section{ANÁLISIS DE LA CONFIABILIDAD DE TODA LA PRUEBA}

Índices de dificultad de los ítems (Estadísticos de los elementos)

\begin{tabular}{|c|c|c|c|}
\hline & Media & Desviación típica & $\mathrm{N} .^{\circ}$ \\
\hline Ítem 1 & ,95 & ,225 & 300 \\
\hline Ítem 2 &, 50 & ,501 & 300 \\
\hline Ítem 3 & 67 & , 470 & 300 \\
\hline Ítem 4 & 36 & 480 & 300 \\
\hline Ítem 5 & ,31 & , 462 & 300 \\
\hline Ítem 6 & ,41 & ,492 & 300 \\
\hline Ítem 7 &, 44 & ,497 & 300 \\
\hline Ítem 8 & ,44 & ,497 & 300 \\
\hline Ítem 9 & ,81 & ,390 & 300 \\
\hline Ítem 10 & 37 & ,484 & 300 \\
\hline Ítem 11 & , 48 &, 500 & 300 \\
\hline Ítem 12 & 44 & ,498 & 300 \\
\hline Ítem 13 & ,58 & ,495 & 300 \\
\hline Ítem 14 & 65 & ,477 & 300 \\
\hline Ítem 15 & ,53 & ,500 & 300 \\
\hline Ítem 16 & ,58 & ,495 & 300 \\
\hline Ítem 17 & ,39 & ,488 & 300 \\
\hline Ítem 18 & 24 & 428 & 300 \\
\hline Ítem 19 & ,43 & , 496 & 300 \\
\hline Ítem 20 & 31 & ,465 & 300 \\
\hline Ítem 21 &, 52 &, 501 & 300 \\
\hline Ítem 22 & ,78 & ,415 & 300 \\
\hline Ítem 23 & ,64 & ,481 & 300 \\
\hline Ítem 24 & ,84 & ,364 & 300 \\
\hline Ítem 25 &, 80 & ,398 & 300 \\
\hline Ítem 26 & ,79 & ,406 & 300 \\
\hline Ítem 27 & ,41 & ,493 & 300 \\
\hline Ítem 28 & ,62 & , 487 & 300 \\
\hline Ítem 29 & ,20 & ,401 & 300 \\
\hline Ítem 30 & ,35 & , 479 & 300 \\
\hline Ítem 31 & ,68 & , 469 & 300 \\
\hline Ítem 32 & ,68 & ,469 & 300 \\
\hline Ítem 33 & ,73 & ,443 & 300 \\
\hline Ítem 34 & ,66 & , 473 & 300 \\
\hline Ítem 35 & ,50 & ,501 & 300 \\
\hline Ítem 36 & ,38 & , 485 & 300 \\
\hline Ítem 37 & ,41 & ,492 & 300 \\
\hline Ítem 38 & ,44 & ,497 & 300 \\
\hline Ítem 39 & ,27 & ,443 & 300 \\
\hline Ítem 40 & ,46 & ,499 & 300 \\
\hline Ítem 41 &, 58 &, 495 & 300 \\
\hline
\end{tabular}

Los ítems sombreados corresponden a los nueve que fueron revisados y mejorados, correspondiendo a otra numeración en la prueba de 41 ítems. 
ANEXO G

ESTADÍSTICOS TOTAL-ELEMENTO

\begin{tabular}{|c|c|c|c|c|}
\hline & $\begin{array}{l}\text { Media de la escala } \\
\text { si se elimina el } \\
\text { elemento }\end{array}$ & $\begin{array}{l}\text { Varianza de la } \\
\text { escala si se elimina } \\
\text { el elemento }\end{array}$ & $\begin{array}{l}\text { Correlación } \\
\text { elemento-total } \\
\text { corregida }\end{array}$ & $\begin{array}{c}\text { Alfa de Cronbach } \\
\text { si se elimina el } \\
\text { elemento }\end{array}$ \\
\hline Ítem 1 & 20,66 & 49,255 & ,277 & ,841 \\
\hline Ítem 2 & 21,10 & 47,819 & ,259 & 839 \\
\hline Ítem 3 & 20,93 & 49,594 & ,208 &, 845 \\
\hline Ítem 4 & 21,25 & 47,707 & ,291 & ,838 \\
\hline Ítem 5 & 21,30 & 48,291 & 212 & 840 \\
\hline Ítem 6 & 21,20 & 47,686 & ,285 & ,839 \\
\hline Ítem 7 & 21,17 & 48,637 & 241 &, 842 \\
\hline Ítem 8 & 21,17 & 47,653 & , 286 & 839 \\
\hline Ítem 9 & 20,79 & 47,944 & ,327 &, 838 \\
\hline Ítem 10 & 21,24 & 47,071 & ,386 &, 836 \\
\hline Ítem 11 & 21,13 & 47,451 & 314 & 838 \\
\hline Ítem 12 & 21,16 & 46,532 & ,454 &, 834 \\
\hline Ítem 13 & 21,03 & 47,782 & 269 & 839 \\
\hline Ítem 14 & 20,95 & 46,767 &, 440 &, 835 \\
\hline Ítem 15 & 21,08 & 47,543 & ,301 &, 838 \\
\hline Ítem 16 & 21,03 & 47,320 & ,338 & 837 \\
\hline Ítem 17 & 21,22 & 47,149 &, 370 &, 836 \\
\hline Ítem 18 & 21,37 & 50,480 & ,231 & ,848 \\
\hline Ítem 19 & 21,17 & 47,548 & ,302 & ,838 \\
\hline Ítem 20 & 21,29 & 47,920 & ,269 & ,839 \\
\hline Ítem 21 & 21,09 & 48,851 & ,209 &, 843 \\
\hline Ítem 22 & 20,83 & 47,816 &, 327 &, 838 \\
\hline Ítem 23 & 20,97 & 48,567 & ,259 &, 842 \\
\hline Ítem 24 & 20,76 & 48,014 & ,341 &, 838 \\
\hline Ítem 25 & 20,80 & 47,476 & ,406 &, 836 \\
\hline Ítem 26 & 20,81 & 47,885 &, 323 &, 838 \\
\hline Ítem 27 & 21,20 & 48,480 & ,267 &, 842 \\
\hline Ítem 28 & 20,99 & 47,495 & ,318 &, 838 \\
\hline Ítem 29 & 21,41 & 47,600 & ,381 & ,836 \\
\hline Ítem 30 & 21,25 & 47,775 & ,281 & ,839 \\
\hline Ítem 31 & 20,93 & 47,256 &, 371 &, 836 \\
\hline Ítem 32 & 20,93 & 46,728 & ,456 &, 834 \\
\hline Ítem 33 & 20,87 & 46,867 & ,462 & 834 \\
\hline Ítem 34 & 20,94 & 46,656 & ,462 &, 834 \\
\hline Ítem 35 & 21,11 & 46,974 & ,384 &, 836 \\
\hline Ítem 36 & 21,23 & 46,699 & ,442 & ,834 \\
\hline Ítem 37 & 21,20 & 46,595 & ,451 & ,834 \\
\hline Ítem 38 & 21,17 & 46,229 & ,502 & ,833 \\
\hline Ítem 39 & 21,34 & 48,968 & ,213 & ,842 \\
\hline Ítem 40 & 21,15 & 46,233 & ,498 & ,833 \\
\hline Ítem 41 & 21,03 & 46,357 &, 484 &, 833 \\
\hline
\end{tabular}


ANEXO H

\section{COMUNALIDADES}

\begin{tabular}{|c|c|c|}
\hline Ítem & Inicial & Extracción \\
\hline Ítem 1 & 1,000 &, 518 \\
\hline Ítem 2 & 1,000 &, 517 \\
\hline Ítem 4 & 1,000 & ,505 \\
\hline Ítem 5 & 1,000 &, 571 \\
\hline Ítem 7 & 1,000 & ,704 \\
\hline Ítem 9 & 1,000 & ,542 \\
\hline Ítem 10 & 1,000 & ,469 \\
\hline Ítem 11 & 1,000 & ,648 \\
\hline Ítem 12 & 1,000 &, 534 \\
\hline Ítem 13 & 1,000 & ,592 \\
\hline Ítem 16 & 1,000 &, 557 \\
\hline Ítem17 & 1,000 & ,436 \\
\hline Ítem 18 & 1,000 & ,483 \\
\hline Ítem 19 & 1,000 & ,492 \\
\hline Ítem 22 & 1,000 & ,568 \\
\hline Ítem 23 & 1,000 &, 527 \\
\hline Ítem 25 & 1,000 & ,622 \\
\hline Ítem 26 & 1,000 &, 503 \\
\hline Ítem 27 & 1,000 &, 563 \\
\hline Ítem 28 & 1,000 & ,495 \\
\hline Ítem 29 & 1,000 &, 540 \\
\hline Ítem 30 & 1,000 & ,483 \\
\hline Ítem 31 & 1,000 & ,672 \\
\hline Ítem 32 & 1,000 &, 547 \\
\hline Ítem 35 & 1,000 &, 524 \\
\hline Ítem 36 & 1,000 &, 570 \\
\hline Ítem 37 & 1,000 & ,508 \\
\hline Ítem 38 & 1,000 &, 524 \\
\hline Ítem 39 & 1,000 & ,644 \\
\hline Ítem 42 & 1,000 &, 585 \\
\hline Ítem 44 & 1,000 & ,611 \\
\hline Ítem 45 & 1,000 & ,573 \\
\hline
\end{tabular}


ANEXO I

VARIANZA TOTAL EXPLICADA

\begin{tabular}{|c|c|c|c|}
\hline \multirow{2}{*}{ Componente } & \multicolumn{3}{|c|}{ Autovalores iniciales } \\
\hline & Total & $\%$ de la varianza & $\%$ acumulado \\
\hline 1 & 5,833 & 18,229 & 18,229 \\
\hline 2 & 1,727 & 5,397 & 23,627 \\
\hline 3 & 1,644 & 5,138 & 28,765 \\
\hline 4 & 1,446 & 4,519 & 33,283 \\
\hline 5 & 1,337 & 4,178 & 37,461 \\
\hline 6 & 1,269 & 3,966 & 41,427 \\
\hline 7 & 1,147 & 3,586 & 45,012 \\
\hline 8 & 1,123 & 3,510 & 48,522 \\
\hline 9 & 1,071 & 3,348 & 51,870 \\
\hline 10 & 1,029 & 3,216 & 55,085 \\
\hline 11 & ,989 & 3,092 & 58,177 \\
\hline 12 & ,953 & 2,977 & 61,154 \\
\hline 13 & ,913 & 2,854 & 64,008 \\
\hline 14 & 878 & 2,745 & 66,753 \\
\hline 15 & 842 & 2,631 & 69,385 \\
\hline 16 &, 792 & 2,476 & 71,861 \\
\hline 17 &, 777 & 2,429 & 74,290 \\
\hline 18 &, 769 & 2,404 & 76,695 \\
\hline 19 &, 722 & 2,255 & 78,950 \\
\hline 20 & ,698 & 2,180 & 81,130 \\
\hline 21 & 685 & 2,142 & 83,272 \\
\hline 22 & ,616 & 1,924 & 85,196 \\
\hline 23 &, 584 & 1,824 & 87,019 \\
\hline 24 &, 549 & 1,716 & 88,735 \\
\hline 25 &, 545 & 1,704 & 90,439 \\
\hline 26 &, 515 & 1,609 & 92,048 \\
\hline 27 &, 508 & 1,588 & 93,636 \\
\hline 28 &, 488 & 1,526 & 95,161 \\
\hline 29 & ,426 & 1,332 & 96,493 \\
\hline 30 &, 403 & 1,260 & 97,754 \\
\hline 31 &, 378 & 1,180 & 98,934 \\
\hline 32 &, 341 & 1,066 & 100,000 \\
\hline
\end{tabular}

Método de extracción: Análisis de Componentes principales. 


\section{ANEXO J}

\section{MATRIZ DE COMPONENTES}

\begin{tabular}{|c|c|c|c|}
\hline & \multicolumn{3}{|c|}{ Componente } \\
\hline & 1 & 2 & 3 \\
\hline Ítem 38 & ,619 &,- 229 &, 125 \\
\hline Ítem 36 &, 582 &,- 303 & \\
\hline Ítem 37 &, 576 &,- 163 & \\
\hline Ítem 29 &, 550 &,- 150 &,- 379 \\
\hline Ítem 13 & ,539 & ,300 &,- 122 \\
\hline Ítem 12 & ,519 & ,198 &,- 144 \\
\hline Ítem 25 &, 510 &,- 235 &,- 341 \\
\hline Ítem 27 &, 503 & &,- 474 \\
\hline Ítem 44 & ,502 &,- 215 & , 127 \\
\hline Ítem 17 & ,488 &, 132 & ,201 \\
\hline Ítem 10 & ,483 & & \\
\hline Ítem 28 & ,461 &,- 125 &,- 427 \\
\hline Ítem 16 &, 451 & ,298 &, 168 \\
\hline Ítem 39 & ,450 &,- 286 & ,273 \\
\hline Ítem 26 & ,445 & &,- 323 \\
\hline Ítem 35 &, 431 &,- 123 & \\
\hline Ítem 18 & ,413 & ,292 &, 134 \\
\hline Ítem 19 & ,402 &, 228 & ,159 \\
\hline Ítem 23 & ,401 & & ,274 \\
\hline Ítem 31 & ,379 &, 159 &,- 281 \\
\hline Ítem 2 & ,347 & & \\
\hline Ítem 45 & ,346 & & ,200 \\
\hline Ítem 30 & ,308 &,- 264 &, 129 \\
\hline Ítem 9 & ,300 &, 162 &, 192 \\
\hline Ítem 1 & ,255 &,- 135 & \\
\hline Ítem 5 & ,255 & ,448 &, 170 \\
\hline Ítem 22 & ,335 & ,409 & ,227 \\
\hline Ítem 11 & ,264 & 391 & \\
\hline Ítem 4 & ,306 &,- 386 &, 170 \\
\hline Ítem 7 & ,235 &, 274 &,- 232 \\
\hline Ítem 32 & ,293 & & ,326 \\
\hline Ítem 42 & ,263 &,- 202 & ,279 \\
\hline
\end{tabular}

Método de extracción: Análisis de componentes principales.

a. 3 componentes extraídos 
ANEXO K

MATRIZ DE CONFIGURACIÓN A

\begin{tabular}{|c|c|c|c|}
\hline & \multicolumn{3}{|c|}{ Componente } \\
\hline & 1 & 2 & 3 \\
\hline Ítem 39 & ,596 & & \\
\hline Ítem 36 & ,574 & & \\
\hline Ítem 38 & ,561 & & \\
\hline Ítem 4 & ,539 & & \\
\hline Ítem 44 & 491 & & \\
\hline Ítem 42 & ,440 & & \\
\hline Ítem 37 & ,427 & & \\
\hline Ítem 30 & ,427 & & \\
\hline Ítem 23 & ,418 & & \\
\hline Ítem 35 & ,350 & & \\
\hline Ítem 45 & ,325 & & \\
\hline Ítem 32 & ,302 & & \\
\hline Ítem 1 & 206 & & \\
\hline Ítem 2 & ,202 & & \\
\hline Ítem 22 & & ,588 & \\
\hline Ítem 5 & & ,568 & \\
\hline Ítem 16 & & ,507 & \\
\hline Ítem 18 & & , 472 & \\
\hline Ítem 11 & & , 471 & \\
\hline Ítem 19 & & , 423 & \\
\hline Ítem 13 & & ,413 & \\
\hline Ítem 17 & & ,388 & \\
\hline Ítem 9 & & ,341 & \\
\hline Ítem 27 & & &,- 695 \\
\hline Ítem 28 & & &,- 633 \\
\hline Ítem 29 & & &,- 632 \\
\hline Ítem 25 & & &,- 579 \\
\hline Ítem 26 & & &,- 525 \\
\hline Ítem 31 & & &,- 451 \\
\hline Ítem 12 & & &,- 391 \\
\hline Ítem 7 & & &,- 331 \\
\hline Ítem 10 & & &,- 281 \\
\hline
\end{tabular}

Método de extracción: Análisis de componentes principales. Metodo de rotación: Normalización Oblimin con Kaiser.

a. La rotación ha convergido en 9 iteraciones. 
ANEXO L

MATRIZ DE ESTRUCTURA

\begin{tabular}{|c|c|c|c|}
\hline & \multicolumn{3}{|c|}{ Componentes (Factores) } \\
\hline & 1 & 2 & 3 \\
\hline Ítem 38 & ,635 & & \\
\hline Ítem 36 & ,629 & & \\
\hline Ítem 39 &, 598 & & \\
\hline Ítem 44 &, 541 & & \\
\hline Ítem 37 &, 522 & & \\
\hline Ítem 4 &, 500 & & \\
\hline Ítem 23 & ,454 & & \\
\hline Ítem 30 & ,421 & & \\
\hline Ítem 42 & ,416 & & \\
\hline Ítem 35 & ,413 & & \\
\hline Ítem 45 &, 365 & & \\
\hline Ítem 32 &, 332 & & \\
\hline Ítem 2 & 277 & & \\
\hline Ítem 1 &, 241 & & \\
\hline Ítem 22 & &, 573 & \\
\hline Ítem 16 & & ,551 & \\
\hline Ítem 5 & &, 530 & \\
\hline Ítem 13 & &, 514 & \\
\hline Ítem 18 & & ,511 & \\
\hline Ítem 17 & & ,470 & \\
\hline Ítem 19 & & ,468 & \\
\hline Ítem 11 & & ,461 & \\
\hline Ítem 9 & & ,368 & \\
\hline Ítem 27 & & &,- 693 \\
\hline Ítem 29 & & &,- 657 \\
\hline Ítem 28 & & &,- 626 \\
\hline Ítem 25 & & &,- 601 \\
\hline Ítem 26 & & &,- 548 \\
\hline Ítem 12 & & &,- 486 \\
\hline Ítem 31 & & &,- 473 \\
\hline Ítem 10 & & &,- 387 \\
\hline Ítem 7 & & &,- 336 \\
\hline
\end{tabular}

Método de rotación: Normalización Oblimin con Kaiser.

Método de extracción: Análisis de componentes principales. 
ANEXO $M$

\section{ANÁLISIS DE LA CONFIABILIDAD DEL FACTOR 1}

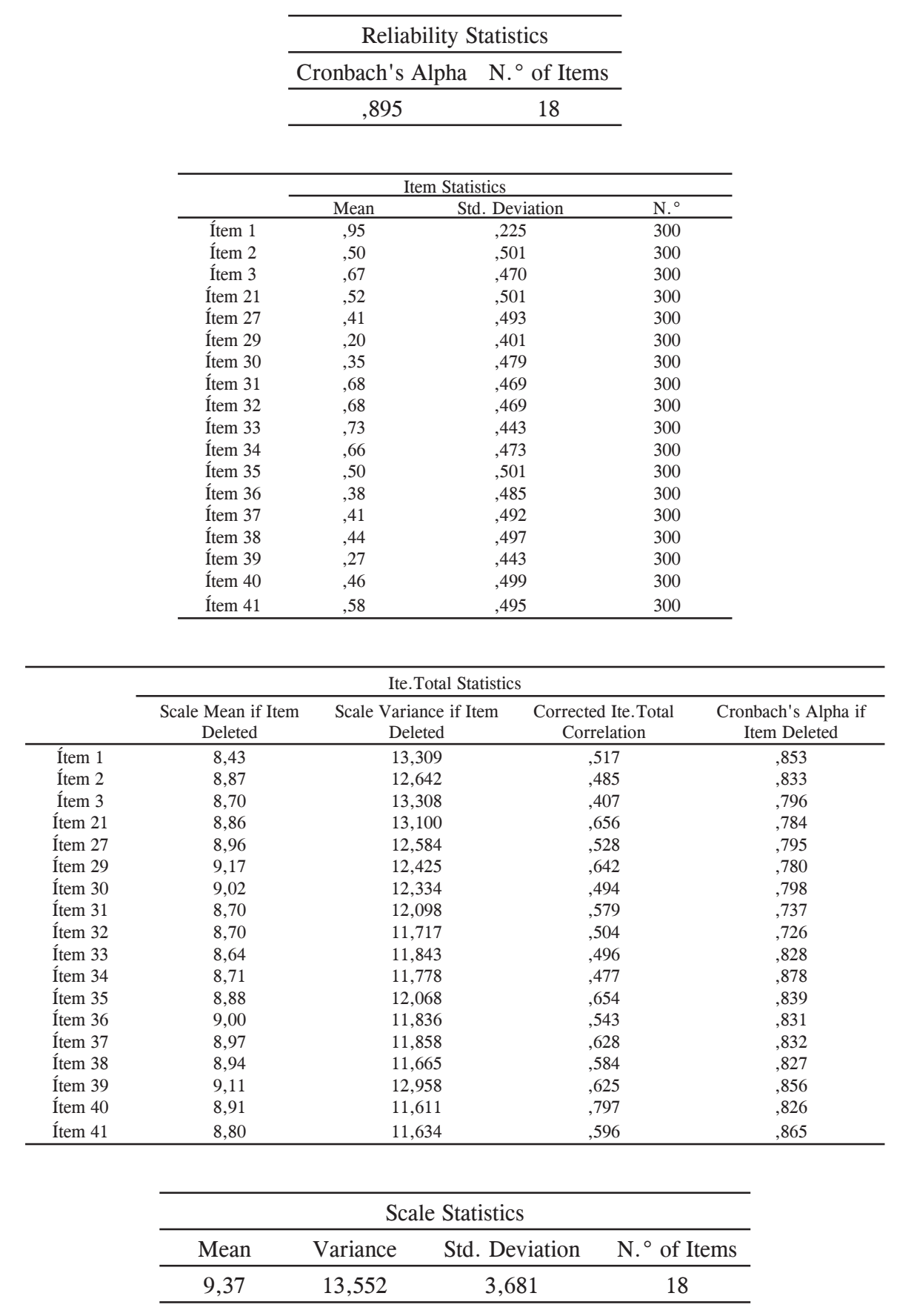


ANÁLISIS DE LA CONFIABILIDAD DEL FACTOR 2

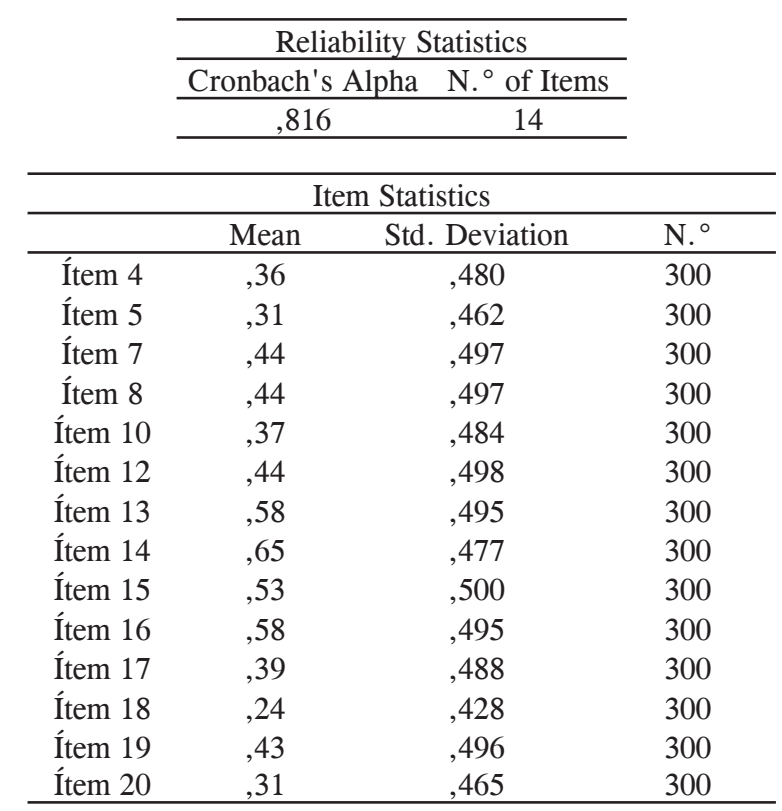

\begin{tabular}{|c|c|c|c|c|}
\hline \multicolumn{5}{|c|}{ Item. total Statistics } \\
\hline & $\begin{array}{c}\text { Scale Mean if Item } \\
\text { Deleted }\end{array}$ & $\begin{array}{l}\text { Scale Variance if } \\
\text { Item Deleted }\end{array}$ & $\begin{array}{l}\text { Corrected Ite.Total } \\
\text { Correlation }\end{array}$ & $\begin{array}{l}\text { Cronbach's Alpha } \\
\text { if Item Deleted }\end{array}$ \\
\hline Ítem 4 & 5,70 & 6,711 &, 522 & ,769 \\
\hline Ítem 5 & 5,75 & 6,976 & 618 & ,789 \\
\hline Ítem 7 & 5,62 & 7,212 & 609 & ,779 \\
\hline Ítem 8 & 5,62 & 6,844 & ,722 & ,765 \\
\hline Ítem 10 & 5,69 & 6,402 & ,541 &, 770 \\
\hline Ítem 12 & 5,62 & 6,291 & ,442 & ,763 \\
\hline Ítem 13 & 5,48 & 6,812 &, 526 & ,762 \\
\hline Ítem 14 & 5,41 & 6,523 & 636 &, 778 \\
\hline Ítem 15 & 5,53 & 6,645 & 420 & ,791 \\
\hline Ítem 16 & 5,48 & 6,598 &, 534 & ,787 \\
\hline Ítem 17 & 5,67 & 6,602 & ,430 & ,786 \\
\hline Ítem 18 & 5,82 & 7,727 & 613 &, 765 \\
\hline Ítem 19 & 5,63 & 6,797 &, 521 & ,762 \\
\hline \multirow[t]{4}{*}{ Ítem 20} & 5,75 & 6,832 & ,625 & ,799 \\
\hline & \multicolumn{4}{|c|}{ Scale Statistics } \\
\hline & Mean & Variance & N. ${ }^{\circ}$ of Ite & \\
\hline & 6,06 & 7,642 & 764 & \\
\hline
\end{tabular}




\section{ANÁLISIS DE LA CONFIABILIDAD DEL FACTOR 3}

\begin{tabular}{|c|c|c|c|}
\hline & \multicolumn{2}{|c|}{ Reliability Statistics } & \\
\hline & Cronbach's Alpha & a $\quad{ }^{\circ}$ of Items & \\
\hline & ,783 & 10 & \\
\hline \multicolumn{4}{|c|}{ Item Statistics } \\
\hline & Mean & d. Deviation & N. ${ }^{\circ}$ \\
\hline Ítem 6 & ,41 & ,492 & 300 \\
\hline Ítem 9 & ,81 & ,390 & 300 \\
\hline Ítem 11 & ,48 & ,500 & 300 \\
\hline Ítem 22 & ,78 & ,415 & 300 \\
\hline Ítem 23 & ,64 & ,481 & 300 \\
\hline Ítem 24 & ,84 & ,364 & 300 \\
\hline Ítem 25 & ,80 & ,398 & 300 \\
\hline Ítem 26 & ,79 & ,406 & 300 \\
\hline Ítem 28 & ,62 & ,487 & 300 \\
\hline
\end{tabular}

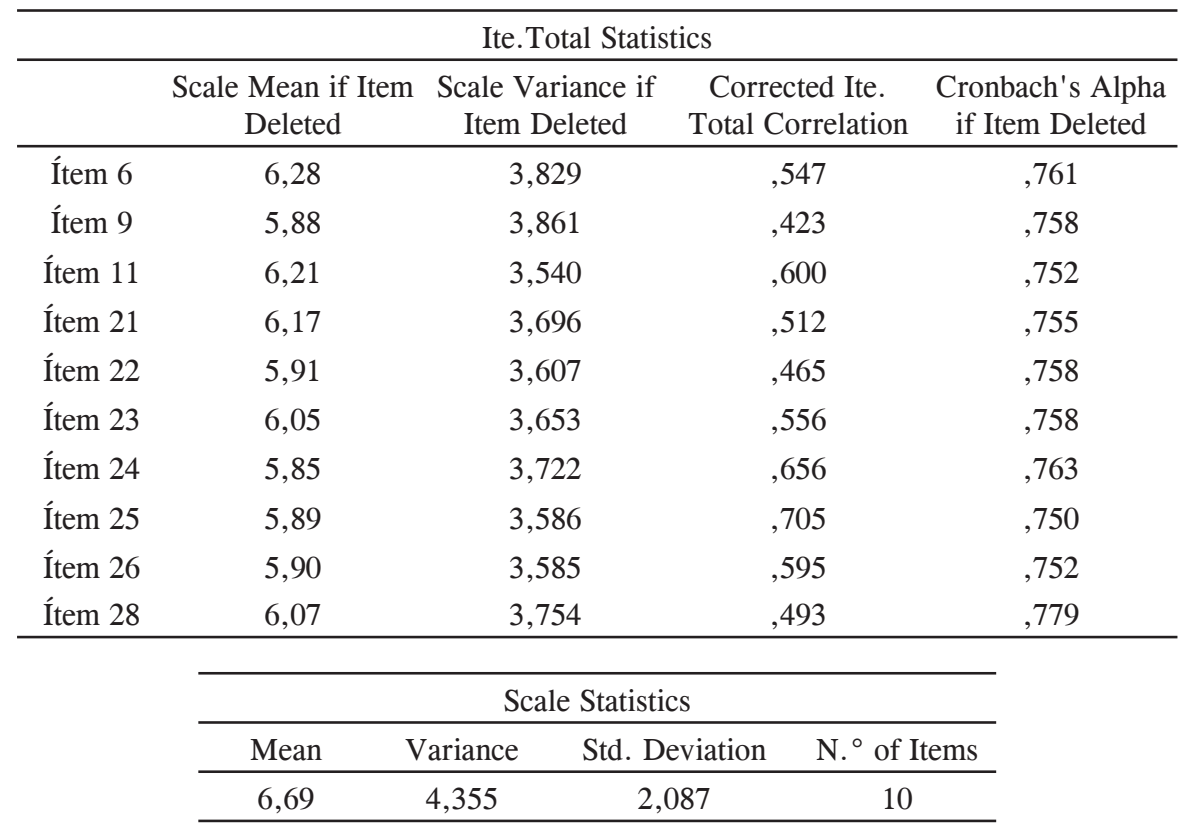

Las operaciones y funciones del pensamiento identificadas son: 


\section{OPERACIONES MENTALES (R. Feuerstein)}

Razonamiento lógico

Pensamiento divergente

Razonamiento silogístico

Razonamiento transitivo

Razonamiento hipotético

Razonamiento analógico

Inferencia lógica

Análisis - Síntesis

Proyección de Relaciones Virtuales

Codificación - Decodificación

Clasificación

Comparación

Transformación mental

Representación mental

Diferenciación

Identificación 


\section{FUNCIONES COGNITIVAS}

\begin{tabular}{|c|c|c|}
\hline F. de Entrada & F. de Elaboración & F. de Salida \\
\hline $\begin{array}{l}\text { 1. Percepción clara y precisa. } \\
\text { 2. Comportamiento exploratorio } \\
\text { sistemático. } \\
\text { 3. Uso de vocabulari.conceptos } \\
\text { apropiados. } \\
\text { 4. Orientación espacial eficiente. } \\
\text { 5. Orientación temporal eficiente. } \\
\text { 6. Constancia, permanencia del } \\
\text { objeto. } \\
\text { 7. Recopilación de datos con } \\
\text { precisión y exactitud. } \\
\text { 8. Considerar dos o más fuentes } \\
\text { de información a la vez. }\end{array}$ & $\begin{array}{l}\text { 1. Percibir el problema y } \\
\text { definirlo con claridad. } \\
\text { 2. Facilidad para distinguir datos } \\
\text { relevantes e irrelevantes. } \\
\text { 3. Ejercitar la conducta } \\
\text { comparativa espontánea. } \\
\text { 4. Amplitud y flexibilidad } \\
\text { mental. } \\
\text { 5. Percepción global (no } \\
\text { episódica de la realidad). } \\
\text { 6. Uso de razonamiento lógico. } \\
\text { 7. Interiorización del propio } \\
\text { comportamiento. } \\
\text { 8. Ejercicio del pensamiento } \\
\text { hipotético diferencial. } \\
\text { 9. Trazar estrategias para } \\
\text { verificar hipótesis. } \\
\text { 10. Conducta planificada. } \\
\text { 11. Elaboración de categorías } \\
\text { cognitivas. } \\
\text { 12. Aplicación de la conducta } \\
\text { sumativa. } \\
\text { 13. Facilidad para establecer } \\
\text { relaciones virtuales. }\end{array}$ & $\begin{array}{l}\text { 1. Comunicación descentralizada } \\
\text { (no egocéntrica). } \\
\text { 2. Proyección de relaciones } \\
\text { virtuales. } \\
\text { 3. Expresión (sin bloqueo) en la } \\
\text { comunicación de respuestas. } \\
\text { 4. Respuestas certeras (sin ensayo } \\
\text { y error). } \\
\text { 5. Uso de instrumentos verbales } \\
\text { adecuados. } \\
\text { 6. Precisión y exactitud en la } \\
\text { comunicación de respuestas. } \\
\text { 7. Eficacia en el transporte visual. } \\
\text { 8. Conducta controlada, no } \\
\text { impulsiva. }\end{array}$ \\
\hline
\end{tabular}

\section{PROCESOS DEL PENSAMIENTO (M. SÁNCHEZ)}

\begin{tabular}{|c|c|c|c|}
\hline P. Básicos & P. de Razonamiento & P. Creativos & P. Superiores \\
\hline $\begin{array}{l}\text { 1. De construcción de } \\
\text { conocimientos: obser- } \\
\text { vación, comparación, } \\
\text { relación y clasificación. } \\
\text { 2. De organización del } \\
\text { conocimiento: ordena- } \\
\text { miento y clasificación } \\
\text { jerárquica. } \\
\text { 3. De integración y juicio } \\
\text { crítico: análisis, síntesis } \\
\text { y evaluación. }\end{array}$ & $\begin{array}{l}\text { 1. Deductivo, inductivo, } \\
\text { hipotético y analógico. }\end{array}$ & $\begin{array}{l}\text { 1. Expansión y contrac- } \\
\text { ción de ideas. } \\
\text { 2. Extensión de campo y } \\
\text { activación cognitiva. } \\
\text { 3. Inventiva. }\end{array}$ & $\begin{array}{l}\text { 1. Procesos directivos } \\
\text { para el manejo de la } \\
\text { información. } \\
\text { 2. Procesos ejecutivos } \\
\text { para el manejo de la } \\
\text { información. } \\
\text { 3. Procesos de adquisi- } \\
\text { ción de conocimientos. } \\
\text { 4. Discernimiento. }\end{array}$ \\
\hline
\end{tabular}

